\title{
酰胺类杂环烯酮缩胺的合成
}

\author{
罗大云崔时胜胡兴梅林 军* 严胜骄* \\ (云南大学化学科学与工程学院 教育部自然资源药物化学重点实验室 昆明 650091)
}

\begin{abstract}
摘要 建立了以杂环烯酮缩胺(HKAs)为原料, 用芳基异氰酸酯为酰化剂, 实现杂环烯酮缩胺选择性酰化. 通过该区域 选择性反应简洁、快速地合成了一系具有潜在药理活性的酰胺类杂环烯酮缩胺化合物的方法. 该方法以六元或七元环 杂环烯酮缩胺 $1 \sim 2$ 与芳基异氧酸酯 4 在 1,4-二氧六环溶剂中室温下合成杂环烯酮缩胺 $\alpha$-碳选择性酰基化产物 $\mathbf{5} \sim \mathbf{6}$, 产 率 90\% 98\%; 而五元环杂环烯酮缩胺 3 与芳基异氰酸酯 4 在 1,4-二氧六环溶剂中碳酸铯催化室温下合成杂环烯酮缩 胺选择性氮酰基化产物 7, 产率 78\% 93\%.
\end{abstract}

关键词 杂环烯酮缩胺; 异氧酸酯; 区域选择性; 酰化反应

\section{Synthesis of Amide Class Heterocyclic Ketene Aminals}

\author{
Luo, Dayun Cui, Shisheng $\quad \mathrm{Hu}$, Xingmei Lin, Jun* Yan, Shengjiao* \\ (Key Laboratory of Medicinal Chemistry for Natural Resource, Ministry of Education, \\ School of Chemical Science and Technology, Yunnan University, Kunming 650091)
}

\begin{abstract}
In this paper, a concise protocol for synthesis of possess potential pharmacological active amide class heterocyclic ketene aminals (HKAs) has been developed, which based on the six or seven-membered HKAs $\mathbf{1} \sim \mathbf{2}$, using aryl isocyanate $\mathbf{4}$ as acylation agent. The regioselective acylation reaction of HKAs in 1,4-dioxane at room temperature gave compounds $\mathbf{5} \sim \mathbf{6}$ with $90 \% \sim 98 \%$ yields. However, the five-membered HKAs 3 and aryl isocyanate 4 reacted in 1,4-dioxane catalyzed by $\mathrm{Cs}_{2} \mathrm{CO}_{3}$ at room temperature to obtain compounds 7 in $78 \% \sim 93 \%$ yields.
\end{abstract}

Keywords heterocyclic ketene aminals; isocyanate; regioselective; acylation reaction

酰胺衍生物是一类重要的杂环化合物, 由于其具有 潜在的药理活性包括抗肺炎、抗肿瘤、抗真菌、抗痢疾、 抗氧化、除草、杀虫等 ${ }^{[1,2]}$, 同时可作为许多有机化合物 及药物分子的合成中间体 ${ }^{[3]}$, 致使其在有机化学、药物 学、植物学以及材料学等方面都有潜在的应用 ${ }^{[4 \sim 6]}$. 酰 胺衍生物中较为重要的一类化合物即苯基腿类化合物 具有优异的除草性能 ${ }^{[7,8]}$, 致使其在植物学以及农业等 领域都有重要的应用价值, 也引起了研究者们的极大关 注. 如除草剂灭草隆、草不隆等均具有苯基嫝结构.

杂环烯酮缩胺 (HKAs) 是一种多功能的合成砌 块 ${ }^{[9 \sim 11]}$, 已广泛用于合成结构多样的杂环化合物, 其中 许多杂环化合物具有优异的多种生理活性, 如抗肿 瘤 ${ }^{[2]} 、$ 除草、杀虫 ${ }^{[13]}$ 、抗焦虑 ${ }^{[14]} 、$ 抗细菌等 ${ }^{[15]}$. 杂环烯 酮缩胺的结构如图 1 所示, 由于结构中含有吸电子基

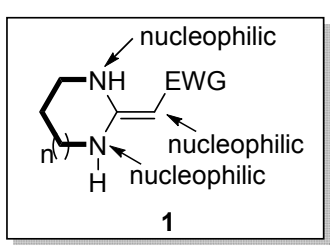

图 1 杂环烯酮缩胺的结构

Figure 1 Structure of heterocyclic ketene aminals

(EWG)和供电性氨基导致碳一碳双键高度极化, 致使 $\alpha$ 碳电子云密度增大, 使得 $\alpha$-碳具有较好亲电性, 可以与 多种亲电试剂发生选择性反应, 如烷基化 ${ }^{[16]}$ 、酰化 ${ }^{[17]}$ 、 糖基化 ${ }^{[18]}$ 、卤化 ${ }^{[19]}$ 、硝化反应 ${ }^{[20]}$ 、芳硫基化 ${ }^{[21]}$. 而通过 与苯基异氧酸酯进行酰化合成具有潜在生物活性的酰 胺类环烯酮缩胺化合物具有重要意义. 从图 1 可知, HKAs 属于缺电子烯胺, 缺电子烯胺的合成多年来一直

* Corresponding authors. E-mail: linjun@ynu.edu.cn; yansj@ynu.edu.cn

Received July 2, 2016; revised August 25, 2016; published online September 8, 2016.

Project supported by the National Natural Science Foundation of China (Nos. 21362042, U1202221, 21662042), the Talent Found in Yunnan Province (No. 2012HB001), and the Excellent Young Talents, Yunnan University (No. XT412003).

国家自然科学基金(Nos. 21362042, U1202221, 21662042)、云南省后备人才(No. 2012HB001)、云南大学青年英才计划(No. XT412003)资助项目. 
备受合成化学家们广泛关注 ${ }^{[22]}$.

从方法学上对杂环烯酮缩胺进行区域选择性酰化 反应具有一定意义; 同时由于杂环烯酮缩胺具有广泛生 物活性, 酰胺类化合物也具有广泛生物活性, 所以通过 杂环烯酮缩胺的区域选择性酰化合成的新的酰胺类杂 环烯酮缩胺将具有潜在的生物活性. 本文以杂环烯酮缩 胺 $1 \sim 3$ 与苯基异氭酸酯 4 为反应原料, 在 1,4-二氧六环 溶剂中室温条件下, 通过酰基化反应简洁、高效区域选 择性合成一系列酰胺类杂环烯酮缩胺酰胺化合物 $5 \sim 6$ 和苯基艮结构的杂环烯酮缩胺化合物 7. 该方法具有原 料易得、合成路线简洁、产率高、反应条件温和等优点. 合成路线见 Scheme 1.

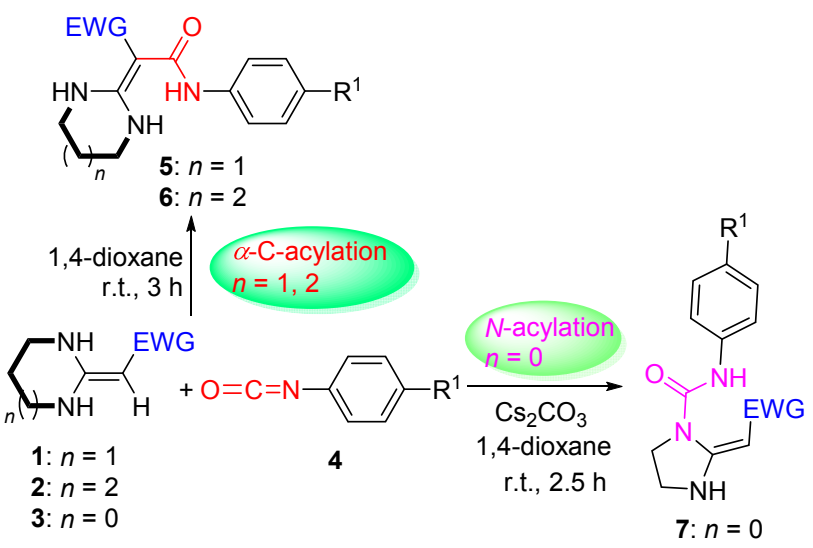

图式 1 目标化合物 5 7 的合成

Scheme 1 Synthesis of target compounds $5 \sim 7$

\section{1 结果与讨论}

\section{1 反应条件的篮选、制备和讨论}

我们以六元环的杂环烯酮缩胺 $1 \mathrm{a}$ 与 4-氯苯基异氰 酸酯(4a)为模板反应, 分别对反应溶剂、催化剂、反应 时间、温度等进行条件篮选与优化，结果见表 1 .

首先, 将杂环烯酮缩胺 $1 \mathbf{a}$ 与 4-氯苯基异㲵酸酯(4a) 按等物质的量溶解于丙酮中, 室温条件下反应 $3 \mathrm{~h}$, 结 果以 $91 \%$ 的收率得目标化合物 $\mathbf{5 a}$ (表 1, Entry 1). 在此基 础上, 笁选了乙腈、乙醇、1,4-二氧六环、甲苯、四氢 呋喃五种溶剂(表 1, Entries 2 6). 结果表明溶剂对反应 收率有较大影响, 其中 1,4-二氧六环为溶剂时以 $96 \%$ 的 收率得目标化合物 $\mathbf{5 a}$ (表 1, Entry 4). 从表 1 可看出, 1,4二氧六环为最佳溶剂. 对催化剂包括 DIPEA, $\mathrm{Et}_{3} \mathrm{~N}$, $\mathrm{K}_{2} \mathrm{CO}_{3}, \mathrm{Cs}_{2} \mathrm{CO}_{3}$ 进行笁选. 结果显示, 催化剂对反应速 率有一定的提高, 但反应的副产物也随之增多, 致使反 应的收率有所下降, 尤其是碳酸铯作为催化剂时反应收 率下降到 63\%(表 1, Entries 10 vs 7 9). 对反应温度进 行筛选. 反应在 $50{ }^{\circ} \mathrm{C}$ 或回流条件下, 温度提高虽可缩 短反应时间, 但收率却有所降低(表 1, Entries 11, 12),
表 1 反应条件的优化 ${ }^{a}$

Table 1 Optimization of reaction conditions
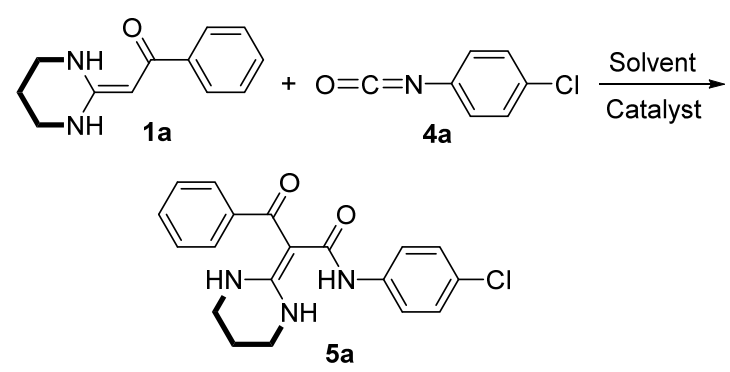

\begin{tabular}{clcccc}
\hline Entry & Solvent & Catalyst & $\mathrm{T}\left({ }^{\circ} \mathrm{C}\right)$ & $\mathrm{Time}^{\mathrm{h}}$ & $\mathrm{Yield}^{b} / \%$ \\
\hline 1 & Acetone & - & r.t. & 3 & 91 \\
2 & Acetonitrile & - & r.t. & 3 & 79 \\
3 & EtOH & - & r.t. & 3 & 21 \\
4 & 1,4-Dioxane & - & r.t. & 3 & 96 \\
5 & Toluene & - & r.t. & 3 & 93 \\
6 & THF & - & r.t. & 3 & 87 \\
7 & 1,4-Dioxane & $\mathrm{DIPEA}^{2}$ & r.t. & 3 & 89 \\
8 & 1,4-Dioxane & $\mathrm{Et}_{3} \mathrm{~N}$ & r.t. & 2 & 94 \\
9 & 1,4-Dioxane & $\mathrm{K}_{2} \mathrm{CO}_{3}$ & r.t. & 2.5 & 81 \\
10 & 1,4-Dioxane & $\mathrm{Cs}_{2} \mathrm{CO}_{3}$ & r.t. & 2 & 63 \\
11 & 1,4-Dioxane & - & 50 & 2.5 & 93 \\
12 & 1,4-Dioxane & - & 110 & 2.5 & 91 \\
13 & 1,4-Dioxane & - & r.t. & $\mathbf{3}$ & $\mathbf{9 7}^{c}$ \\
14 & 1,4-Dioxane & - & r.t. & 3 & $94^{d}$ \\
\hline
\end{tabular}

${ }^{a}$ The reaction was performed with 1a $(1 \mathrm{mmol}), 4 a(1 \mathrm{mmol})$ and the solvent $(15 \mathrm{~mL}) .{ }^{b}$ Isolated yields. ${ }^{c}$ The reaction was performed with $\mathbf{1 a}(1 \mathrm{mmol}), \mathbf{4 a}$ $(1.1 \mathrm{mmol})$, and the solvent $(15 \mathrm{~mL}) .{ }^{d}$ The reaction was performed with 1a (1.1 mmol), 4a (1 mmol), and the solvent $(15 \mathrm{~mL})$.

确定室温是最佳温度. 最后, 对原料的物质的量比进行 筛选. 将 HKAs (1a) 与 4-氯苯基异氰酸酯(4a)的物质的 量比由 $1: 1$ 调整为 $1: 1.1$ 时, 反应进行较完全且目标 化合物的收率也较高(表 1, Entries 4 vs 13 vs 14). 最终 确定最佳反应条件为: $1 \mathrm{a}$ 与 $\mathbf{4 a}$ 的物质的量比为 $1: 1.1$, 溶剂为 1,4-二氧六环，室温条件下搅拌 $3 \mathrm{~h}$.

在最佳条件基础上探索该反应的普适性. 我们选择 供电子的甲基、甲氧基和吸电子的氟、氯、溴取代的 HKAs, 邻位和对位取代的 HKAs, 六元和七元环的 HKAs 作为反应底物进行测试. 研究结果表明, 对于六 元环的 HKAs 而言, 供电子基团 $(\mathrm{Me}, \mathrm{MeO})$ 比吸电子基 团(F、Cl、Br)取代的 HKAs 有更高的收率(表 2, Entries $1 \sim 6)$. 此外, 对位取代较邻位取代的 HKAs 有较高的收 率(表 2, Entries 4 vs 7; Entries 5 vs 8). 噻吩杂环烯酮缩 胺同样以优异的收率得到目标产物(表 2, Entry 9). 七元 环 HKAs 为底物时同样以优异的收率得到目标化合物 6(表 2, Entries 14 20). 从反应收率看, 给电子基略高 于吸电子基 HKAs(表 2, Entries 15 vs 16 vs 17).

此外, 我们用不同取代的苯基异氰酸酯 $\mathbf{4 b} \sim \mathbf{4 d}$ 为 反应底物与六～七元杂环烯酮缩胺反应. 结果表明, 不 同取代苯基异氰酸酯对产物收率有一定影响。供电子 
表 2 酰胺类杂环烯酮缩胺 $5 \sim 6$ 的制备 ${ }^{a}$

Table 2 Preparation of amide class heterocyclic ketene aminals $5 \sim 6$

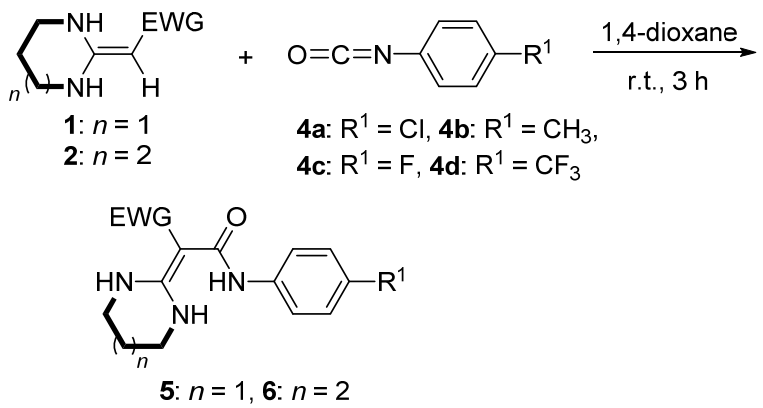

\begin{tabular}{|c|c|c|c|c|}
\hline Entry & 1/2 (EWG/n) & 4 & $5 / 6$ & Yield $^{b} / \%$ \\
\hline 1 & 1a $(\mathrm{PhCO} / 1)$ & $4 a$ & $5 \mathbf{5 a}$ & 97 \\
\hline 2 & $\mathbf{1 b}\left(4-\mathrm{MeOC}_{6} \mathrm{H}_{4} \mathrm{CO} / 1\right)$ & $4 a$ & $5 \mathbf{b}$ & 95 \\
\hline 3 & 1c $\left(4-\mathrm{MeC}_{6} \mathrm{H}_{4} \mathrm{CO} / 1\right)$ & $4 a$ & $5 c$ & 96 \\
\hline 4 & 1d $\left(4-\mathrm{FC}_{6} \mathrm{H}_{4} \mathrm{CO} / 1\right)$ & $4 a$ & 5d & 92 \\
\hline 5 & 1e $\left(4-\mathrm{ClC}_{6} \mathrm{H}_{4} \mathrm{CO} / 1\right)$ & $4 a$ & $5 e$ & 90 \\
\hline 6 & 1f $\left(4-\mathrm{BrC}_{6} \mathrm{H}_{4} \mathrm{CO} / 1\right)$ & $4 a$ & $5 f$ & 92 \\
\hline 7 & $\lg \left(2-\mathrm{FC}_{6} \mathrm{H}_{4} \mathrm{CO} / 1\right)$ & $4 a$ & $5 g$ & 91 \\
\hline 8 & 1h $\left(2-\mathrm{ClC}_{6} \mathrm{H}_{4} \mathrm{CO} / 1\right)$ & $4 a$ & $5 \mathrm{~h}$ & 90 \\
\hline 9 & $\mathbf{1 i}\left(\mathrm{C}_{4} \mathrm{H}_{3} \mathrm{SCO} / 1\right)$ & $4 a$ & $5 \mathbf{i}$ & 97 \\
\hline 10 & $\mathbf{1} \mathbf{j}\left(\mathrm{NO}_{2} / 1\right)$ & $4 a$ & $5 \mathbf{j}$ & 95 \\
\hline 11 & $1 \mathbf{a}(\mathrm{PhCO} / 1)$ & $4 b$ & $5 \mathbf{k}$ & 98 \\
\hline 12 & $\mathbf{1 a}(\mathrm{PhCO} / 1)$ & $4 c$ & 51 & 96 \\
\hline 13 & $\mathbf{1 a}(\mathrm{PhCO} / 1)$ & 4d & $5 \mathrm{~m}$ & 96 \\
\hline 14 & $\mathbf{2 a}(\mathrm{PhCO} / 2)$ & $4 a$ & $6 \mathbf{a}$ & 94 \\
\hline 15 & 2b $\left(4-\mathrm{MeC}_{6} \mathrm{H}_{4} \mathrm{CO} / 2\right)$ & $4 a$ & $6 \mathbf{b}$ & 97 \\
\hline 16 & 2c $\left(4-\mathrm{FC}_{6} \mathrm{H}_{4} \mathrm{CO} / 2\right)$ & $4 a$ & $6 c$ & 92 \\
\hline 17 & 2d $\left(4-\mathrm{ClC}_{6} \mathrm{H}_{4} \mathrm{CO} / 2\right)$ & $4 a$ & 6d & 94 \\
\hline 18 & $\mathbf{2 d}\left(4-\mathrm{ClC}_{6} \mathrm{H}_{4} \mathrm{CO} / 2\right)$ & $4 b$ & $6 e$ & 96 \\
\hline 19 & 2d $\left(4-\mathrm{ClC}_{6} \mathrm{H}_{4} \mathrm{CO} / 2\right)$ & $4 c$ & $6 f$ & 95 \\
\hline 20 & $2 d\left(4-\mathrm{ClC}_{6} \mathrm{H}_{4} \mathrm{CO} / 2\right)$ & 4d & $6 \mathrm{~g}$ & 93 \\
\hline
\end{tabular}

${ }^{a}$ The reaction was performed with 1 or $2(1 \mathrm{mmol}), 4(1.1 \mathrm{mmol})$ and the solvent $(15 \mathrm{~mL}) .{ }^{b}$ Isolated yields based on HKAs $\mathbf{1}$ or $\mathbf{2}$.

基苯基异氰酸酯比吸电子基苯基异氧酸酯有更高的收 率(表 2, Entries 11 vs 1 vs 12 vs 13, Entries 18 vs 17 vs 19 vs 20).

当五元环杂环烯酮缩胺 3a 与 4-氯苯基异氰酸酯 (4a)在上述同样条件下反应, 以 $73 \%$ 的收率获得杂环烯 酮缩胺的氮酰基化产物 7a. 由于反应的收率还不够理 想, 我们对溶剂、温度、催化剂等进行了篮选优化. 首 先, $3 \mathbf{a}$ 与 $4 \mathbf{a}$ 的物质的量比为 $1: 1.1$, 选择丙酮、乙腈等 溶剂(表 3, Entries 1 7). 结果发现 1,4-二氧六环仍然是 较为理想的溶剂; 在催化剂篮选时发现, 随着催化剂碱 性的增强, 目标化合物的收率有所提高, 但当我们使用 碱性较强的叔丁醇钾和氢化钠作为催化剂时, 薄层色谱 (TLC)检测表明该反应副产物增多导致目标化合物的收 率降低(表 3, Entries 4 vs $8 \sim 13$ ). 温度的提高使得反应 更为复杂, 收率大为下降(表 3, Entries 11 vs 15). 据此, 反应的最佳条件是以 1,4 -二氧六环为溶剂, 碳酸铯为催
化剂，室温下反应 $2.5 \mathrm{~h}$. 同时，我们也利用该条件重新 对六元环和七元环 HKAs 进行测试, 发现该条件下仅有 少量的氮酰基化产物产生, 主产物依然是发生在 HKAs 的 $\alpha$-碳的酰基化产物.

\section{表 3 反应条件的优化 ${ }^{a}$}

Table 3 Optimization of reaction conditions

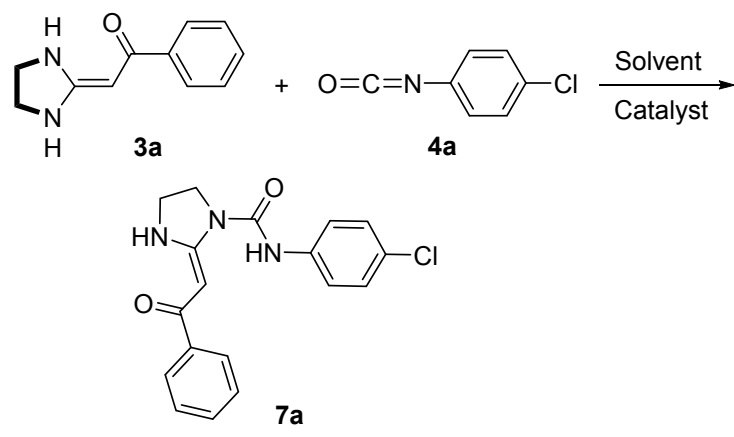

\begin{tabular}{|c|c|c|c|c|c|}
\hline Entry & Solvent & Catalyst & $t /{ }^{\circ} \mathrm{C}$ & Time/h & Yield $^{b} / \%$ \\
\hline 1 & Acetone & - & r.t. & 3 & 71 \\
\hline 2 & Acetonitrile & - & r.t. & 3 & 42 \\
\hline 3 & EtOH & - & r.t. & 3 & 13 \\
\hline 4 & 1,4-Dioxane & - & r.t. & 3 & 73 \\
\hline 5 & Toluene & - & r.t. & 3 & 68 \\
\hline 6 & Tetrahydrofuran & - & r.t. & 3 & 56 \\
\hline 7 & Chloroform & - & r.t. & 3 & 61 \\
\hline 8 & 1,4-Dioxane & DIPEA & r.t. & 3 & 75 \\
\hline 9 & 1,4-Dioxane & $\mathrm{Et}_{3} \mathrm{~N}$ & r.t. & 3 & 77 \\
\hline 10 & 1,4-Dioxane & $\mathrm{K}_{2} \mathrm{CO}_{3}$ & r.t. & 2.5 & 83 \\
\hline 11 & 1,4-Dioxane & $\mathrm{Cs}_{2} \mathrm{CO}_{3}$ & r.t. & 2.5 & 91 \\
\hline 12 & 1,4-Dioxane & $t$-BuOK & r.t. & 1.5 & 79 \\
\hline 13 & 1,4-Dioxane & $\mathrm{NaH}$ & r.t. & 1.5 & 72 \\
\hline 14 & 1,4-Dioxane & - & 80 & 1.5 & 32 \\
\hline 15 & 1,4-Dioxane & $\mathrm{Cs}_{2} \mathrm{CO}_{3}$ & 80 & 1.5 & 38 \\
\hline
\end{tabular}

${ }^{a}$ The reaction was performed with $\mathbf{3 a}(1 \mathrm{mmol}), \mathbf{4 a}(1.1 \mathrm{mmol})$, catalyst $(0.5$ mmol) and the solvent $(15 \mathrm{~mL}){ }^{b}$ Isolated yields based on HKAs 3a.

在获得最佳反应条件基础上，我们考察不同取代的 杂环烯酮缩胺和苯基异氰酸酯的反应情况. 从表 4 可看 出，供电子基 HKAs 反应的收率高于吸电基 $\mathrm{HKAs}$ (表 4, Entries 2、3 vs 4 vs 5 vs 6). 而苯基异氧酸酯苯环上的吸 电子基有利于收率(表 4, Entries 1 vs 10～12).

\section{2 反应选择性}

对于六元环 HKAs 进行 $\alpha$-C 选择性酰化反应，而五 元环 HKAs 进行氮原子的选择性酰化反应, 这主要受空 间位阻作用. 烯胺类化合物受位阻作用而进行选择性反 应已有文献报道 ${ }^{[23 ~ 25]}$. 我们利用高斯软件分别计算六 元环 HKAs 1a 和五元环 HKAs 3a 的最优构象 (图 2). 从 图 2 可以看出化合物 $\mathbf{1 a}$ 呈 $\mathrm{U}$ 型结构, 而化合物 $\mathbf{3 a}$ 呈近 似直线型分子. 可看出, 六元环 HKAs 1a 的 $\alpha-\mathrm{C}(\mathrm{C} 3)$ 的 位阻比五元环 HKAs 3a 的 $\alpha-C$ (C3)位阻小; 反之, 六元 
表 4 酰胺类杂环烯酮缩胺酰胺 7 的制备

Table 4 Preparation of amide class heterocyclic ketene aminals $7^{a}$

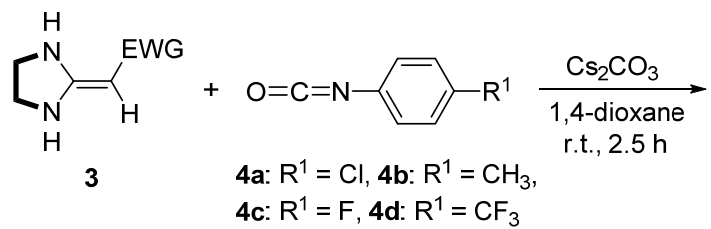<smiles>[R14]C=C1NCCN1C(=O)Nc1ccc([R16])cc1</smiles>

\begin{tabular}{clccc}
\hline Entry & \multicolumn{1}{c}{$\mathbf{3}(\mathrm{EWG})$} & $\mathbf{4}$ & $\mathbf{7}$ & Yield $^{b} \%$ \\
\hline 1 & $\mathbf{3 a}(\mathrm{PhCO})$ & $\mathbf{4 a}$ & $\mathbf{7 a}$ & 91 \\
2 & $\mathbf{3 b}\left(4-\mathrm{MeOC}{ }_{6} \mathrm{H}_{4} \mathrm{CO}\right)$ & $\mathbf{4 a}$ & $\mathbf{7 b}$ & 90 \\
3 & $\mathbf{3 c}\left(4-\mathrm{MeC}_{6} \mathrm{H}_{4} \mathrm{CO}\right)$ & $\mathbf{4 a}$ & $\mathbf{7 c}$ & 89 \\
4 & $\mathbf{3 d}\left(4-\mathrm{FC}_{6} \mathrm{H}_{4} \mathrm{CO}\right)$ & $\mathbf{4 a}$ & $\mathbf{7 d}$ & 85 \\
5 & $\mathbf{3 e}\left(4-\mathrm{ClC}_{6} \mathrm{H}_{4} \mathrm{CO}\right)$ & $\mathbf{4 a}$ & $\mathbf{7 e}$ & 87 \\
6 & $\mathbf{3 f}\left(4-\mathrm{BrC}_{6} \mathrm{H}_{4} \mathrm{CO}\right)$ & $\mathbf{4 a}$ & $\mathbf{7 f}$ & 78 \\
7 & $\mathbf{3 g}\left(2-\mathrm{ClC}_{6} \mathrm{H}_{4} \mathrm{CO}\right)$ & $\mathbf{4 a}$ & $\mathbf{7 g}$ & 79 \\
8 & $\mathbf{3 h}\left(\mathrm{C}_{4} \mathrm{H}_{3} \mathrm{~S}\right)$ & $\mathbf{4 a}$ & $\mathbf{7 h}$ & 91 \\
9 & $\mathbf{3 i}\left(\mathrm{NO}_{2}\right)$ & $\mathbf{4 a}$ & $\mathbf{7 i}$ & 90 \\
10 & $\mathbf{3 a}(\mathrm{PhCO})$ & $\mathbf{4 b}$ & $\mathbf{7 j}$ & 85 \\
11 & $\mathbf{3 a}(\mathrm{PhCO})$ & $\mathbf{4 c}$ & $\mathbf{7 k}$ & 91 \\
12 & $\mathbf{3 a}(\mathrm{PhCO})$ & $\mathbf{4 d}$ & $\mathbf{7 l}$ & 93 \\
\hline
\end{tabular}

${ }^{a}$ The reaction was performed with $3(1 \mathrm{mmol}), 4(1.1 \mathrm{mmol}), \mathrm{CsCO}_{3}(0.5$ mmol) and the solvent $(15 \mathrm{~mL}) .{ }^{b}$ Isolated yields based on HKAs 3 .

环 HKAs $1 \mathbf{a}$ 的氮原子 $(\mathrm{N} 4)$ 的位阻比五元环 HKAs $\mathbf{3 a}$ 的 氮原子(N4)位阻大; 在化合物 $1 \mathbf{a}$ 和 $\mathbf{3 a}$ 中羰基氧均与氮 原子 $(\mathrm{N} 1)$ 形成分子内氢键, 导致 $\mathrm{N} 1$ 位很难参加反应. 由上可知, 是位阻作用导致六元环杂环烯酮缩胺 1 在与 苯基异氰酸酯 4 反应时优先进行的是 $\alpha-\mathrm{C}$ 酰化反应; 而 五元环杂环烯酮缩胺 3 在与苯基异氧酸酯 4 反应时优先 进行的是氮原子(N4)的酰化反应.
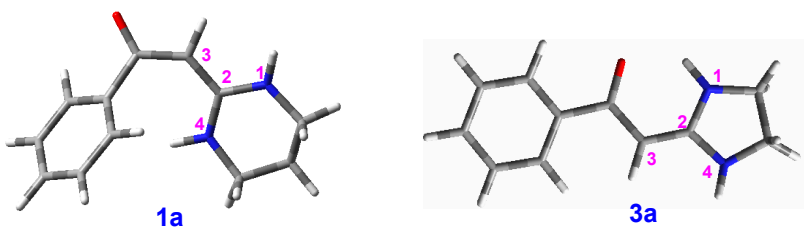

图 2 杂环烯酮缩胺 1a 和 3a 的最优构象

Figure 2 Optimal conformations of heterocyclic ketene aminals 1a and 3a

\section{3 反应机理}

我们以化合物 $5 \mathbf{a}$ 为例阐明该反应的可能机理: 首 先杂环烯酮缩胺 1a 对 4-氯苯基异氰酸酯(4a)加成得中 间体 8 , 中间体 8 经过亚胺一烯胺 ${ }^{[11 a]}$ 互变形成中间体 $\mathbf{9}$, 化合物 9 经过酮一烯醇互变形成化合物 5a. 反应机理如
Scheme 2 所示.
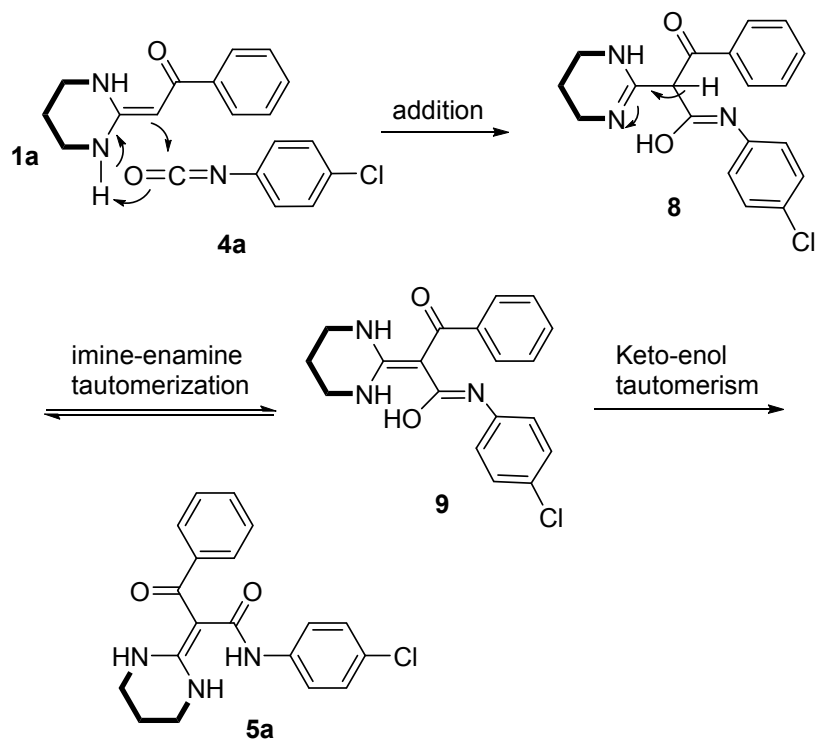

图式 2 推测的反应机理

Scheme 2 Proposed mechanism

\section{2 结论}

总之，本文成功建立了区域选择性简洁、高效合成 具有潜在生物活性的酰胺类杂环烯酮缩胺化合物库的 方法. 该方法以六元或七元环杂环烯酮缩胺 $1 \sim 2$ 与异 氭酸酯 4 在 1,4-二氧六环溶剂中室温下反应 $3 \mathrm{~h}$ 后以优 异的收率(90\% 98\%)得到杂环烯酮缩胺 $\alpha$-碳酰基化产 物 5 6; 而五元环杂环烯酮缩胺 3 与异氰酸酯 4 在 1,4二氧六环溶剂中, 用碳酸铯为催化剂, 室温下反应 $2.5 \mathrm{~h}$ 后以良好的收率(78\% 93\%)得杂环烯酮缩胺氮酰基化 产物 7. 该方法具有操作简便、合成路线简洁、产率高、 区域选择性好等优点. 所合成的酰胺类杂环烯酮缩胺化 合物具有潜在的生物活性, 这将为今后进一步进行活性 笁选打下基础.

\section{3 实验部分}

\section{1 仪器和试剂}

控温型电磁搅拌器; 傅里叶红外光谱仪(Thermo Nicolet Avatar 360 型); 高分辨质谱仪(Agilent CL/Msd TOF); 核磁共振仪 Bruck DRX300 $\left({ }^{1} \mathrm{H}: 300 \mathrm{MHz},{ }^{13} \mathrm{C}: 75\right.$ $\mathrm{MHz})$ 或 DRX500 $\left({ }^{1} \mathrm{H}: 500 \mathrm{MHz},{ }^{13} \mathrm{C}: 125 \mathrm{MHz}\right)$; 熔点仪 (XT-4A 控温型显微熔点测定仪). 试剂用分析纯或化学 纯(无水硫酸钠干燥处理). $\mathrm{GF}_{254}$ 高效薄层层析板及柱层 析硅胶(200～300 目，青岛海洋化工厂). 反应原料 1 3 按文献[26]制备.

\section{2 化合物 $5 \sim 6$ 的合成方法}

称取 $1 \mathrm{mmol}$ 杂环烯酮缩胺 1 2 加入到 $25 \mathrm{~mL}$ 圆底 
烧瓶中, 再加入 $15 \mathrm{~mL}$ 溶剂摚拌溶解. 摚拌下, 缓慢加 入 $1.1 \mathrm{mmol}$ 苯基异氰酸酯 4 , 室温下搅拌 $3 \mathrm{~h}, \mathrm{TLC}$ 监测 直至杂环烯酮缩胺反应完全后, 停止反应. 密封圆底烧 瓶后于冰箱内静置约 $0.5 \mathrm{~h}$ 后, 反应液中有固体析出, 抽 滤. 滤液经过减压蒸馏并留少量液体接着加入一定量的 石油醚, 析出固体, 再抽滤. 合并两次抽滤所得固体用 适量丙酮重结晶得目标产物 $\mathbf{5} \sim \mathbf{6}$.

$N$-(4-氯苯基)-3-氧代-3-苯基-2-[四氢嘧啶-2 $(1 H)$-亚 基]丙酰胺(5a): 得 $345 \mathrm{mg}$, 白色固体, 产率 97\%. m.p. 186.3 188.3 ${ }^{\circ} \mathrm{C} ;{ }^{1} \mathrm{H}$ NMR $\left(300 \mathrm{MHz}, \mathrm{CDCl}_{3}\right) \delta: 1.97 \sim$ $2.05\left(\mathrm{~m}, 2 \mathrm{H}, \mathrm{CH}_{2}\right), 3.41 \sim 3.47\left(\mathrm{~m}, 4 \mathrm{H}, 2 \mathrm{CH}_{2}\right), 6.83(\mathrm{~d}, J=$ 8.4Hz, 2H, ArH), 6.94 (br, 1H, NH), 7.11 (d, $J=8.4 \mathrm{~Hz}$, $2 \mathrm{H}, \operatorname{ArH}), 7.36 \sim 7.42(\mathrm{~m}, 3 \mathrm{H}, \mathrm{ArH}), 7.58(\mathrm{~d}, J=3.9 \mathrm{~Hz}$, $2 \mathrm{H}, \mathrm{ArH}), 10.21$ (br, $2 \mathrm{H}, 2 \mathrm{NH}) ;{ }^{13} \mathrm{C}$ HMR $(75 \mathrm{MHz}$, $\left.\mathrm{CDCl}_{3}\right) \delta$ : 19.7, 38.2, 38.2, 90.8, 120.8, 127.7, 128.3, 128.5, 128.7, 130.1, 136.9, 143.1, 160.1, 170.0, 189.5; IR (KBr) $v$ : 3282, 2869, 1628, 1488, 1367, 1321, 1284, 1245, 1196, 1145, 1094, 1046, 826, 777, 749, 702, $507 \mathrm{~cm}^{-1}$; HRMS (TOF $\mathrm{ES}^{+}$) calcd for $\mathrm{C}_{19} \mathrm{H}_{17} \mathrm{ClN}_{3} \mathrm{O}_{2}[\mathrm{M}-\mathrm{H}]^{-}$ 354.1015, found 354.1013 .

$\mathrm{N}$-(4-氯苯基)-3-(4-甲氧基苯基)-3-氧代-2-[四氢嘧 啶-2(1H)-亚基]丙酰胺(5b): 得 $366 \mathrm{mg}$, 白色固体, 产率 95\%. m.p. $168.0 \sim 170.0{ }^{\circ} \mathrm{C} ;{ }^{1} \mathrm{H}$ NMR (300 MHz, $\mathrm{CDCl}_{3}$ ) $\delta: 1.97 \sim 2.03\left(\mathrm{~m}, 2 \mathrm{H}, \mathrm{CH}_{2}\right), 3.40 \sim 3.46\left(\mathrm{~m}, 4 \mathrm{H}, 2 \mathrm{CH}_{2}\right)$, $3.82\left(\mathrm{~s}, 3 \mathrm{H}, \mathrm{OCH}_{3}\right), 6.86 \sim 7.15(\mathrm{~m}, 7 \mathrm{H}, \mathrm{ArH}+\mathrm{NH})$, $7.53 \sim 7.59$ (m, 2H, ArH), 10.15 (br, 2H, 2NH); ${ }^{13} \mathrm{C} \mathrm{HMR}$ $\left(75 \mathrm{MHz}, \mathrm{CDCl}_{3}\right) \delta: 19.8,38.3,38.3,55.4,90.2,113.9$, $120.9,128.2,128.6,129.9,135.3,137.1,160.1,161.2$, 170.3, 189.1; IR (KBr) v: 3427, 1622, 1602, 1492, 1362, 1321, 1251, 1168, 1032, $782 \mathrm{~cm}^{-1}$; HRMS (TOF ES ${ }^{+}$) calcd for $\mathrm{C}_{20} \mathrm{H}_{19} \mathrm{ClN}_{3} \mathrm{O}_{3}[\mathrm{M}-\mathrm{H}]^{-}$384.1120, found 384.1121 .

$N$-(4-氯苯基)-3-氧代 -2-[四氢嘧啶 - $2(1 H)$ - 亚 基]-3-(对甲苯基)丙酰胺 $(\mathbf{5 c})$ : 得 $355 \mathrm{mg}$, 白色固体, 产 率 96\%. m.p. 190.1 192.1 ${ }^{\circ} \mathrm{C} ;{ }^{1} \mathrm{H}$ NMR $(300 \mathrm{MHz}$, $\left.\mathrm{CDCl}_{3}\right) \delta: 1.97 \sim 2.03\left(\mathrm{~m}, 2 \mathrm{H}, \mathrm{CH}_{2}\right), 2.36\left(\mathrm{~s}, 3 \mathrm{H}, \mathrm{CH}_{3}\right)$, $3.41 \sim 3.47\left(\mathrm{~m}, 4 \mathrm{H}, 2 \mathrm{CH}_{2}\right), 6.83 \sim 7.17(\mathrm{~m}, 7 \mathrm{H}, \mathrm{ArH}+$ $\mathrm{NH}), 7.44 \sim 7.50(\mathrm{~m}, 2 \mathrm{H}, \mathrm{ArH}), 10.20(\mathrm{br}, 2 \mathrm{H}, 2 \mathrm{NH}) ;{ }^{13} \mathrm{C}$ HMR (75 MHz, $\left.\mathrm{CDCl}_{3}\right) \delta: 19.7,21.4,38.2,38.2,90.6$, $120.8,127.8,128.2,128.5,129.3,137.1,140.2,140.3$, $160.1,170.2,189.7$; IR (KBr) v: 3286, 1626, 1591, 1526, $1491,1401,1361,1320,1245,1172,831,780 \mathrm{~cm}^{-1}$; HRMS (TOF ES ${ }^{+}$) calcd for $\mathrm{C}_{20} \mathrm{H}_{19} \mathrm{ClN}_{3} \mathrm{O}_{2}[\mathrm{M}-\mathrm{H}]^{-}$ 368.1171 , found 368.1170 .

$N$-(4-氯苯基)-3-(4-氟苯基)-3-氧代-2-[四氢嘧啶-
2(1H)-亚基]丙酰胺 $(\mathbf{5 d})$ : 得 $343 \mathrm{mg}$, 白色固体, 产率 92\%. m.p. 176.9 178.9 ${ }^{\circ} \mathrm{C} ;{ }^{1} \mathrm{H}$ NMR (300 MHz, $\mathrm{CDCl}_{3}$ ) $\delta: 1.97-2.03\left(\mathrm{~m}, 2 \mathrm{H}, \mathrm{CH}_{2}\right), 3.40 \sim 3.46\left(\mathrm{~m}, 4 \mathrm{H}, 2 \mathrm{CH}_{2}\right)$, $6.90 \sim 7.17(\mathrm{~m}, 7 \mathrm{H}, \mathrm{ArH}+\mathrm{NH}), 7.57 \sim 7.63(\mathrm{~m}, 2 \mathrm{H}, \mathrm{ArH})$, 10.08 (br, 2H, NH); ${ }^{13} \mathrm{C}$ HMR (75 MHz, $\mathrm{CDCl}_{3}$ ) $\delta: 19.7$, $38.3,38.3,90.7,115.5$ (d, $J=21.0 \mathrm{~Hz}), 120.7,128.7,130.1$ (d, $J=8.3 \mathrm{~Hz}), 136.8,139.1,159.9,169.9,187.9$; IR (KBr) $v: 33284,1621,1598,1491,1402,1319,1232,1092,831$, $783 \mathrm{~cm}^{-1}$; HRMS (TOF ES ${ }^{+}$) calcd for $\mathrm{C}_{19} \mathrm{H}_{16} \mathrm{ClFN}_{3} \mathrm{O}_{2}$ $[\mathrm{M}-\mathrm{H}]^{-}$372.0921, found 372.0923.

$N, 3$-双(4-氯苯基)-3-氧代-2-[四氢嘧啶-2(1H)-亚基] 丙酰胺 (5e): 得 $351 \mathrm{mg}$, 白色固体, 产率 90\%. m.p. 232.4 234.4 ${ }^{\circ} \mathrm{C} ;{ }^{1} \mathrm{H}$ NMR (300 MHz, $\left.\mathrm{CDCl}_{3}\right) \delta: 1.87 \sim$ $1.95\left(\mathrm{~m}, 2 \mathrm{H}, \mathrm{CH}_{2}\right), 3.33\left(\mathrm{t}, J=4.5 \mathrm{~Hz}, 4 \mathrm{H}, 2 \mathrm{CH}_{2}\right), 6.81(\mathrm{~d}$, $J=8.7 \mathrm{~Hz}, 2 \mathrm{H}, \mathrm{ArH}), 7.05(\mathrm{~d}, J=8.7 \mathrm{~Hz}, 2 \mathrm{H}, \mathrm{ArH}), 7.22$ (d, $J=8.4 \mathrm{~Hz}, 2 \mathrm{H}, \mathrm{ArH}), 7.43(\mathrm{~d}, J=8.1 \mathrm{~Hz}, 2 \mathrm{H}, \mathrm{ArH})$, $9.96(\mathrm{br}, 2 \mathrm{H}, 2 \mathrm{NH}) ;{ }^{13} \mathrm{C}$ HMR $\left(75 \mathrm{MHz}, \mathrm{CDCl}_{3}\right) \delta: 19.6$, $38.3,38.3,90.9,120.8,128.6,128.7,128.7,129.3,136.0$, 136.8, 141.3, 159.9, 169.8, 187.7; IR (KBr) v: 3425, 1621, 1591, 1526, 1491, 1400, 1320, 1203, 1174, 1090, 1014, $827,780 \mathrm{~cm}^{-1}$; HRMS (TOF ES ${ }^{+}$) calcd for $\mathrm{C}_{19} \mathrm{H}_{16} \mathrm{Cl}_{2^{-}}$ $\mathrm{N}_{3} \mathrm{O}_{2}[\mathrm{M}-\mathrm{H}]^{-}$388.0625, found 388.0622.

3-(4-澳苯基)- $N$-(4-氯苯基)-3-氧代-2-[四氢嘧啶2(1H)-亚基]丙酰胺 $(\mathbf{5 f})$ : 得 $399 \mathrm{mg}$, 白色固体, 产率 92\%. m.p. 179.2 181.2 ${ }^{\circ} \mathrm{C} ;{ }^{1} \mathrm{H}$ NMR (300 MHz, $\mathrm{CDCl}_{3}$ ) $\delta: 1.97 \sim 2.03\left(\mathrm{~m}, 2 \mathrm{H}, \mathrm{CH}_{2}\right), 3.40 \sim 3.46\left(\mathrm{~m}, 4 \mathrm{H}, 2 \mathrm{CH}_{2}\right)$, $6.91(\mathrm{~d}, J=7.8 \mathrm{~Hz}, 2 \mathrm{H}, \mathrm{ArH}), 7.03(\mathrm{br}, 1 \mathrm{H}, \mathrm{NH}), 7.16$ (d, $J=7.8 \mathrm{~Hz}, 2 \mathrm{H}, \mathrm{ArH}), 7.44 \sim 7.50(\mathrm{~m}, 4 \mathrm{H}, \mathrm{ArH}), 10.03(\mathrm{br}$, $2 \mathrm{H}, 2 \mathrm{NH}) ;{ }^{13} \mathrm{C}$ HMR $\left(75 \mathrm{MHz}, \mathrm{CDCl}_{3}\right) \delta: 19.6,38.3,38.3$, $90.9,120.8,124.3,128.6,128.7,129.5,131.7,136.8$, 141.7, 159.9, 169.8, 187.7; IR (KBr) $v: 3426,3298,2964$, $1620,1594,1524,1490,1400,1364,1322,1245,1204$, $1173,1092,1010,828,779,765 \mathrm{~cm}^{-1}$; HRMS (TOF ES ${ }^{+}$) calcd for $\mathrm{C}_{19} \mathrm{H}_{16} \mathrm{BrClN}_{3} \mathrm{O}_{2}[\mathrm{M}-\mathrm{H}]^{-}$432.0119, found 432.0118 .

$N$-(4-氯苯基)-3-(2-氟苯基)-3-氧代-2-[四氢嘧啶$2(1 H)$-亚基]丙酰胺 $(\mathbf{5 g})$ : 得 $340 \mathrm{mg}$, 白色固体, 产率 91\%. m.p. $154.2 \sim 156.2{ }^{\circ} \mathrm{C} ;{ }^{1} \mathrm{H}$ NMR (300 MHz, $\mathrm{CDCl}_{3}$ ) $\delta: 1.89 \sim 1.96\left(\mathrm{~m}, 2 \mathrm{H}, \mathrm{CH}_{2}\right), 3.32 \sim 3.38\left(\mathrm{~m}, 4 \mathrm{H}, 2 \mathrm{CH}_{2}\right)$, $6.80 \sim 6.86$ (m, 2H, ArH), $6.92(\mathrm{t}, J=9 \mathrm{~Hz}, 1 \mathrm{H}, \mathrm{ArH}), 7.03$ (d, $J=8.4 \mathrm{~Hz}, 2 \mathrm{H}, \mathrm{ArH}), 7.11(\mathrm{t}, J=7.5 \mathrm{~Hz}, 1 \mathrm{H}, \mathrm{ArH})$, $7.19 \sim 7.24(\mathrm{~m}, 1 \mathrm{H}, \mathrm{ArH}), 7.46(\mathrm{t}, J=6.9 \mathrm{~Hz}, 1 \mathrm{H}, \mathrm{ArH})$, 10.18 (br, $2 \mathrm{H}, 2 \mathrm{NH}) ;{ }^{13} \mathrm{C}$ HMR $\left(75 \mathrm{MHz}, \mathrm{CDCl}_{3}\right) \delta: 19.5$, $38.2,38.2,93.1,116.2(\mathrm{~d}, J=22.5 \mathrm{~Hz}), 120.8,124.8$, $128.5,128.7,129.5,130.9,131.2$ (d, $J=8.3 \mathrm{~Hz}), 136.7$, 
156.9, $160.1(\mathrm{~d}, J=22.5 \mathrm{~Hz}), 169.5,182.6$; IR $(\mathrm{KBr}) v$ : 3431, 1619, 1492, 1451, 1399, 1366, 1321, 1241, 1094, $827,772 \mathrm{~cm}^{-1}$; HRMS (TOF $\mathrm{ES}^{+}$) calcd for $\mathrm{C}_{19} \mathrm{H}_{16} \mathrm{Cl}-$ $\mathrm{FN}_{3} \mathrm{O}_{2}[\mathrm{M}-\mathrm{H}]^{-}$372.0921, found 372.0919.

3-(2-氯苯基)- $N$-(4-氯苯基)-3-氧代-2-[四氢嘧啶2(1H)-亚基]丙酰胺 $(\mathbf{5 h})$ : 得 $351 \mathrm{mg}$, 白色固体, 产率 90\%. m.p. $136.0 \sim 138.0{ }^{\circ} \mathrm{C} ;{ }^{1} \mathrm{H}$ NMR $\left(300 \mathrm{MHz}, \mathrm{CDCl}_{3}\right)$ $\delta: 1.87 \sim 1.93\left(\mathrm{~m}, 2 \mathrm{H}, \mathrm{CH}_{2}\right), 3.31 \sim 3.37\left(\mathrm{~m}, 4 \mathrm{H}, 2 \mathrm{CH}_{2}\right)$, 6.68 (br, 1H, NH), $6.78 \sim 6.84$ (m, 2H, ArH), 7.01 (d, $J=$ $8.4 \mathrm{~Hz} 2 \mathrm{H}, \mathrm{ArH}), 7.12 \sim 7.17(\mathrm{~m}, 2 \mathrm{H}, \mathrm{ArH}), 7.27 \sim 7.33$ (m, 2H, ArH), 10.24 (br, 2H, 2NH); ${ }^{13} \mathrm{C}$ HMR $(75 \mathrm{MHz}$, $\left.\mathrm{CDCl}_{3}\right) \delta: 19.4,38.2,38.2,92.7,120.6,127.6,128.2$, $128.4,128.6,129.9,130.2,136.7,142.6,160.0,169.2$, 185.6; IR (KBr) v: 3427, 2969, 1619, 1492, 1399, 1367, 1320, 1238, 1200, 1092, 1056, 828, $775 \mathrm{~cm}^{-1}$; HRMS (TOF $\mathrm{ES}^{+}$) calcd for $\mathrm{C}_{19} \mathrm{H}_{16} \mathrm{Cl}_{2} \mathrm{~N}_{3} \mathrm{O}_{2}[\mathrm{M}-\mathrm{H}]^{-} 388.0625$, found 388.0627.

$N$-(4-氯苯基)-3-氧代 -2-[四氢嘧啶 - $2(1 H)$ - 亚 基]-3-(噻吩-2-基)丙酰胺(5i): 得 $350 \mathrm{mg}$, 黄色固体, 产 率 97\%. m.p. 182.3 184.3 ${ }^{\circ} \mathrm{C} ;{ }^{1} \mathrm{H}$ NMR $(300 \mathrm{MHz}$, DMSO- $\left.d_{6}\right) \delta: 1.81 \sim 1.87\left(\mathrm{~m}, 2 \mathrm{H}, \mathrm{CH}_{2}\right), 3.28 \sim 3.34(\mathrm{~m}$, $\left.4 \mathrm{H}, 2 \mathrm{CH}_{2}\right), 6.92(\mathrm{t}, J=4.1 \mathrm{~Hz}, 1 \mathrm{H}, \mathrm{ArH}), 7.11(\mathrm{~d}, J=3.0$ $\mathrm{Hz}, 1 \mathrm{H}, \mathrm{ArH}), 7.24(\mathrm{~d}, J=8.7 \mathrm{~Hz}, 2 \mathrm{H}, \mathrm{ArH}), 7.47 \sim 7.53$ (m, 3H, ArH), 9.31 (br, 2H, 2NH), $11.12(\mathrm{br}, 1 \mathrm{H}, \mathrm{NH}) ;{ }^{13} \mathrm{C}$ HMR (75 MHz, DMSO- $\left.d_{6}\right) \delta: 18.7,38.2,38.2,91.8,120.2$, $125.2,126.3,126.7,128.3,128.3,139.3,147.9,159.9$, 167.7, 175.0; IR (KBr) v: 3431, 1619, 1492, 1451, 1399, 1366, 1321, 1241, 1094, 827, $772 \mathrm{~cm}^{-1}$; HRMS (TOF $\mathrm{ES}^{+}$) calcd for $\mathrm{C}_{17} \mathrm{H}_{15} \mathrm{ClN}_{3} \mathrm{O}_{2} \mathrm{~S}[\mathrm{M}-\mathrm{H}]^{-}$360.0579, found 360.0579 .

$N$-(4-氯苯基)-2-硝基-2-[四氢嘧啶-2 $(1 H)$-亚基] 乙酰 胺 $(\mathbf{5 j})$ : 得 $281 \mathrm{mg}$, 白色固体, 产率 95\%. m.p. 206.7 $208.7{ }^{\circ} \mathrm{C} ;{ }^{1} \mathrm{H}$ NMR (300 MHz, DMSO- $\left.d_{6}\right) \delta: 1.81 \sim 1.89$ (m, $\left.2 \mathrm{H}, \mathrm{CH}_{2}\right), 3.36 \sim 3.42\left(\mathrm{~m}, 4 \mathrm{H}, 2 \mathrm{CH}_{2}\right), 7.34(\mathrm{~d}, J=8.7$ $\mathrm{Hz}, 2 \mathrm{H}, \mathrm{ArH}), 7.63$ (d, J=8.7 Hz, 2H, ArH), 9.85 (br, 2H, $2 \mathrm{NH}), 11.76$ (br, $1 \mathrm{H}, \mathrm{NH}) ;{ }^{13} \mathrm{C}$ HMR (75 MHz, DMSO- $d_{6}$ ) $\delta$ : 17.8, 38.4, 38.4, 108.7, 121.2, 126.7, 128.6, 137.6, 155.5, 161.4; IR (KBr) v: 3208, 3088, 2879, 1626, 1582, 1534, 1444, 1363, 1316, 1277, 1157, 1099, 830, 771, 511 $\mathrm{cm}^{-1}$; HRMS (TOF ES ${ }^{+}$) calcd for $\mathrm{C}_{12} \mathrm{H}_{12} \mathrm{ClN}_{4} \mathrm{O}_{3}[\mathrm{M}-$ H] ${ }^{-}$295.0603, found 295.0603.

3-氧代-3-苯基-2-[四氢嘧啶-2 $(1 H$ )-亚基]- $N$-(对甲苯 基)丙酰胺(5k): 得 $328 \mathrm{mg}$, 白色固体, 产率 98\%. m.p. 200.9 202.9 ${ }^{\circ} \mathrm{C}$; ${ }^{1} \mathrm{H}$ NMR (300 MHz, $\left.\mathrm{CDCl}_{3}\right) \delta: 1.99 \sim$ $2.03\left(\mathrm{~m}, 2 \mathrm{H}, \mathrm{CH}_{2}\right), 2.26\left(\mathrm{~s}, 3 \mathrm{H}, \mathrm{CH}_{3}\right), 3.43 \sim 3.47(\mathrm{~m}, 4 \mathrm{H}$, $\left.2 \mathrm{CH}_{2}\right), 6.76(\mathrm{~d}, J=8.1 \mathrm{~Hz}, 2 \mathrm{H}, \mathrm{ArH}), 6.97(\mathrm{~d}, J=7.8 \mathrm{~Hz}$, $2 \mathrm{H}, \mathrm{ArH}), 7.37 \sim 7.41(\mathrm{~m}, 3 \mathrm{H}, \mathrm{ArH}), 7.58 \sim 7.62(\mathrm{~m}, 2 \mathrm{H}$, ArH), 10.33 (br, 2H, 2NH); ${ }^{13} \mathrm{C}$ HMR ( $\left.75 \mathrm{MHz}, \mathrm{CDCl}_{3}\right) \delta$ : $19.7,20.8,38.2,38.2,90.9,120.0,127.7,128.7,129.1$, $129.9,133.2,135.6,143.2,160.1,170.1,189.3 \mathrm{~cm}^{-1}$; HRMS (TOF ES ${ }^{+}$) calcd for $\mathrm{C}_{20} \mathrm{H}_{21} \mathrm{~N}_{3} \mathrm{O}_{2}[\mathrm{M}+\mathrm{H}]^{+}$ 336.1707, found 336.1710 .

$N$-(4-氟苯基)-3-氧代-3-苯基-2-[四氢嘧啶-2(1H)-亚 基]丙酰胺(51): 得 $325 \mathrm{mg}$, 白色固体, 产率 96\%. m.p. $204.5 \sim 206.5{ }^{\circ} \mathrm{C} ;{ }^{1} \mathrm{H}$ NMR (300 MHz, $\left.\mathrm{CDCl}_{3}\right) \delta: 2.00 \sim$ $2.04\left(\mathrm{~m}, 2 \mathrm{H}, \mathrm{CH}_{2}\right), 3.43 \sim 3.47\left(\mathrm{~m}, 4 \mathrm{H}, 2 \mathrm{CH}_{2}\right), 6.80 \sim 6.86$ (m, $4 \mathrm{H}, \mathrm{ArH}), 7.37 \sim 7.41(\mathrm{~m}, 3 \mathrm{H}, \mathrm{ArH}), 7.57 \sim 7.61(\mathrm{~m}$, $2 \mathrm{H}, \mathrm{ArH}), 10.24$ (br, 2H, 2NH); ${ }^{13} \mathrm{C}$ HMR $(75 \mathrm{MHz}$, $\left.\mathrm{CDCl}_{3}\right) \delta: 19.7,38.2,38.2,90.7,115.0,115.3,121.6(\mathrm{~d}$, $J=7.5 \mathrm{~Hz}), 127.7,128.7,130.0,134.2,143.2,159.6$ (d, $J=240.7 \mathrm{~Hz}), 160.1,160.7,170.1,189.4$; IR (KBr) $v$ : $3425,3248,3059,2924,2871,1626,1590,1536,1503$, $1445,1366,1322,1208,1145,834,776,735,700 \mathrm{~cm}^{-1}$; HRMS (TOF ES ${ }^{+}$) calcd for $\mathrm{C}_{19} \mathrm{H}_{18} \mathrm{FN}_{3} \mathrm{O}_{2}[\mathrm{M}+\mathrm{H}]^{+}$ 340.1456, found 340.1460 .

3 -氧代-3-苯基-2-[四氢嘧啶-2 $(1 H)$-亚基]- $N$-[4-(三氟 甲基)苯基]丙酰胺 $(\mathbf{5 m})$ : 得 $373 \mathrm{mg}$, 白色固体, 产率 96\%. m.p. $211.7 \sim 213.7{ }^{\circ} \mathrm{C} ;{ }^{1} \mathrm{H}$ NMR (300 $\mathrm{MHz}, \mathrm{CDCl}_{3}$ ) $\delta: 2.00 \sim 2.05\left(\mathrm{~m}, 2 \mathrm{H}, \mathrm{CH}_{2}\right), 3.44 \sim 3.48\left(\mathrm{~m}, 4 \mathrm{H}, 2 \mathrm{CH}_{2}\right)$, $7.02(\mathrm{~d}, J=8.4 \mathrm{~Hz}, 2 \mathrm{H}, \mathrm{ArH}), 7.18$ (br, 1H, NH), 7.37 7.43 (m, 5H, ArH), 7.58 7.61 (m, 2H, ArH), 10.21 (br, $2 \mathrm{H}, 2 \mathrm{NH}) ;{ }^{13} \mathrm{C}$ HMR $\left(75 \mathrm{MHz}, \mathrm{CDCl}_{3}\right) \delta: 19.7,38.3,38.3$, $90.8,118.9,124.7 \sim 125.8(\mathrm{~m}), 127.7,128.8,130.2,141.5$, 143.0, 160.2, 170.1, 189.9; IR (KBr) v: 3422, 3293, 3066, 2931, 2882, 1634, 1618, 1598, 1512, 1472, 1445, 1364, 1321, 1258, 1163, 1068, 870, 841, 776, $705 \mathrm{~cm}^{-1}$; HRMS (TOF $\mathrm{ES}^{+}$) calcd for $\mathrm{C}_{20} \mathrm{H}_{18} \mathrm{~F}_{3} \mathrm{~N}_{3} \mathrm{O}_{2}[\mathrm{M}+\mathrm{H}]^{+}$390.1424, found 390.1425 .

$\mathrm{N}$-(4-氯苯基)-2-(1,3-安定-2-亚基)-3-氧代-3-苯基丙 酰胺 (6a): 得 $347 \mathrm{mg}$, 白色固体, 产率 94\%. m.p. $174.5 \sim 176.5{ }^{\circ} \mathrm{C} ;{ }^{1} \mathrm{H}$ NMR $\left(300 \mathrm{MHz}, \mathrm{CDCl}_{3}\right) \delta: 1.64 \sim$ $1.70\left(\mathrm{~m}, 4 \mathrm{H}, 2 \mathrm{CH}_{2}\right), 3.04 \sim 3.10\left(\mathrm{~m}, 4 \mathrm{H}, 2 \mathrm{CH}_{2}\right), 7.22 \sim$ 7.61 (m, 9H, ArH), 8.71 (br, 2H, NH), 9.88 (br, 1H, NH); ${ }^{13} \mathrm{C}$ HMR (75 MHz, $\mathrm{CDCl}_{3}$ ) $\delta: 27.3,27.3,45.9,45.9,93.4$, $121.4,127.8,128.1,128.7,128.7,130.4,137.4,143.1$, 169.0, 170.3, 191.5; IR (KBr) v: 3300, 3059, 2946, 1632, 1492, 1438, 1399, 1343, 1281, 1244, 1213, 1132, 1087, 1011, 821, 766, 703, 665, $507 \mathrm{~cm}^{-1}$; HRMS (TOF ES ${ }^{+}$) calcd for $\mathrm{C}_{20} \mathrm{H}_{19} \mathrm{ClN}_{3} \mathrm{O}_{2}[\mathrm{M}-\mathrm{H}]^{-}$368.1171, found 368.1174 . 
$N$-(4-氯苯基)-2-(1,3-安定-2-亚基)-3-氧代-3-(对甲苯 基)丙酰胺 $(\mathbf{6 b})$ : 得 $372 \mathrm{mg}$, 白色固体, 产率 97\%. m.p. $180.1 \sim 182.1{ }^{\circ} \mathrm{C} ;{ }^{1} \mathrm{H}$ NMR $\left(300 \mathrm{MHz}, \mathrm{CDCl}_{3}\right) \delta: 1.62 \sim$ $1.68\left(\mathrm{~m}, 4 \mathrm{H}, 2 \mathrm{CH}_{2}\right), 2.37\left(\mathrm{~s}, 3 \mathrm{H}, \mathrm{CH}_{3}\right), 3.03 \sim 3.09(\mathrm{~m}, 4 \mathrm{H}$, $\left.2 \mathrm{CH}_{2}\right), 7.18 \sim 7.32(\mathrm{~m}, 6 \mathrm{H}, \mathrm{ArH}), 7.46 \sim 7.52(\mathrm{~m}, 2 \mathrm{H}$, $\mathrm{ArH}), 8.67$ (br, 2H, 2NH), 10.06 (br, 1H, NH); ${ }^{13} \mathrm{C} \mathrm{HMR}$ $\left(75 \mathrm{MHz}, \mathrm{CDCl}_{3}\right) \delta: 21.4,27.4,27.4,45.9,45.9,93.1$, $121.4,128.0,128.6,129.2,137.6,140.2,140.8,169.0$, 170.4, 191.6; IR (KBr) v: 3426, 3313, 2944, 1628, 1528, 1492, 1438, 1399, 1345, 1211, 1088, 1008, 829, $789 \mathrm{~cm}^{-1}$; HRMS (TOF ES ${ }^{+}$) calcd for $\mathrm{C}_{21} \mathrm{H}_{21} \mathrm{ClN}_{3} \mathrm{O}_{2}[\mathrm{M}-\mathrm{H}]^{-}$ 382.1328 , found 382.1329 .

$\mathrm{N}$-(4-氯苯基)-2-(1,3-安定-2-亚基)-3-(4-氟苯基)-3氧代丙酰胺 $(6 \mathrm{c})$ : 得 $356 \mathrm{mg}$, 白色固体, 产率 $92 \%$. m.p. $168.4 \sim 170.4{ }^{\circ} \mathrm{C} ;{ }^{1} \mathrm{H}$ NMR $\left(300 \mathrm{MHz}, \mathrm{CDCl}_{3}\right) \delta: 1.65 \sim$ $1.71\left(\mathrm{~m}, 4 \mathrm{H}, 2 \mathrm{CH}_{2}\right), 3.08 \sim 3.14\left(\mathrm{~m}, 4 \mathrm{H}, 2 \mathrm{CH}_{2}\right), 7.05 \sim$ $7.26(\mathrm{~m}, 6 \mathrm{H}, \mathrm{ArH}), 7.59 \sim 7.66(\mathrm{~m}, 2 \mathrm{H}, \mathrm{ArH}), 8.77(\mathrm{br}, 2 \mathrm{H}$, $2 \mathrm{NH}), 9.65$ (br, $1 \mathrm{H}, \mathrm{NH}) ;{ }^{13} \mathrm{C} \mathrm{HMR}\left(75 \mathrm{MHz}, \mathrm{CDCl}_{3}\right) \delta$ : 27.3, 27.3, 45.9, 45.9, 93.2, 115.5 (d, $J=21.8 \mathrm{~Hz}), 121.3$, 128.3, 128.7, 130.2 (d, $J=8.3 \mathrm{~Hz}), 137.3,139.1,165.6$, 168.9, 170.0, 189.9; IR (KBr) v: 3429, 3308, 3044, 2934, 2853, 1629, 1599, 1528, 1492, 1400, 1344, 1283, 1221, 1154, 1131, 1090, 1011, 849, 826, 790, $609 \mathrm{~cm}^{-1}$; HRMS $\left(\mathrm{TOF} \mathrm{ES}^{+}\right.$) calcd for $\mathrm{C}_{20} \mathrm{H}_{18} \mathrm{ClFN}_{3} \mathrm{O}_{2}[\mathrm{M}-\mathrm{H}]^{-}$386.1077, found 386.1075 .

$N, 3$-双 (4-氯苯基)-2-(1,3-安定-2-亚基)-3-氧代丙酰 胺(6d): 得 $380 \mathrm{mg}$, 白色固体, 产率 94\%. m.p. 198.6 $200.6{ }^{\circ} \mathrm{C} ;{ }^{1} \mathrm{H}$ NMR $\left(300 \mathrm{MHz}, \mathrm{CDCl}_{3}\right) \delta: 1.65 \sim 1.71(\mathrm{~m}$, $\left.4 \mathrm{H}, 2 \mathrm{CH}_{2}\right), 3.07 \sim 3.14\left(\mathrm{~m}, 4 \mathrm{H}, 2 \mathrm{CH}_{2}\right), 7.21 \sim 7.30(\mathrm{~m}, 4 \mathrm{H}$, ArH), 7.37 (d, $J=8.1 \mathrm{~Hz}, 2 \mathrm{H}, \mathrm{ArH}), 7.55(\mathrm{~d}, J=8.1 \mathrm{~Hz}$, $2 \mathrm{H}, \mathrm{ArH}), 8.72$ (br, $2 \mathrm{H}, 2 \mathrm{NH}), 9.75$ (br, $1 \mathrm{H}, \mathrm{NH}) ;{ }^{13} \mathrm{C}$ HMR (75 MHz, $\left.\mathrm{CDCl}_{3}\right) \delta: 27.3,27.3,45.9,45.9,93.3$, $121.3,128.3,128.8,128.8,129.4,136.4,137.3,141.4$, 168.8, 169.9, 189.7; IR (KBr) v: 3428, 3306, 2930, 1625, 1532, 1491, 1438, 1345, 1282, 1192, 1090, 1012, 844, 789 $\mathrm{cm}^{-1}$; HRMS (TOF ES ${ }^{+}$) calcd for $\mathrm{C}_{20} \mathrm{H}_{18} \mathrm{Cl}_{2} \mathrm{~N}_{3} \mathrm{O}_{2}$ [M$\mathrm{H}]^{-}$402.0782, found 402.0781 .

3-(4-氯苯基)-2-(1,3-安定-2-亚基)-3-氧代- $N$-(对甲苯 基)丙酰胺(6e): 得 $368 \mathrm{mg}$, 白色固体, 产率 96\%. m.p. $204.4 \sim 206.4{ }^{\circ} \mathrm{C} ;{ }^{1} \mathrm{H}$ NMR (300 MHz, DMSO- $d_{6}$ ) $\delta$ : $1.50 \sim 1.54\left(\mathrm{~m}, 4 \mathrm{H}, 2 \mathrm{CH}_{2}\right), 2.21\left(\mathrm{~s}, 3 \mathrm{H}, \mathrm{CH}_{3}\right), 2.95 \sim 2.99$ (m, 4H, $\left.2 \mathrm{CH}_{2}\right), 7.01(\mathrm{~d}, J=8.1 \mathrm{~Hz}, 2 \mathrm{H}, \mathrm{ArH}), 7.32 \sim 7.43$ (m, 6H, ArH), 8.85 (br, 2H, 2NH), $11.21(\mathrm{br}, 1 \mathrm{H}, \mathrm{NH}) ;{ }^{13} \mathrm{C}$ HMR (75 MHz, DMSO- $d_{6}$ ) $\delta: 20.3,26.7,26.7,44.9,44.9$, $93.9,119.0,127.5,128.9,129.3,130.8,133.2,137.4$,
142.5, 167.3, 169.1, 186.5; IR (KBr) v: 3425, 3297, 3037, 2945, 1626, 1591, 1514, 1488, 1439, 1404, 1360, 1248, 1173, 1109, 1072, 1040, 845, 814, $789 \mathrm{~cm}^{-1}$; HRMS (TOF $\mathrm{ES}^{+}$) calcd for $\mathrm{C}_{21} \mathrm{H}_{22} \mathrm{ClN}_{3} \mathrm{O}_{2}[\mathrm{M}+\mathrm{H}]^{+} 383.1473$, found 383.1473 .

3-(4-氯苯基)-2-(1,3-安定-2-亚基)- $N$-(4-氟苯基)-3氧代丙酰胺(6f): 得 $368 \mathrm{mg}$, 白色固体, 产率 $95 \%$. m.p. $214.6 \sim 216.6{ }^{\circ} \mathrm{C} ;{ }^{1} \mathrm{H}$ NMR (300 MHz, DMSO- $d_{6}$ ) $\delta$ : $1.51 \sim 1.55\left(\mathrm{~m}, 4 \mathrm{H}, 2 \mathrm{CH}_{2}\right), 2.96 \sim 3.00\left(\mathrm{~m}, 4 \mathrm{H}, 2 \mathrm{CH}_{2}\right)$, $7.04(\mathrm{t}, J=8.9 \mathrm{~Hz}, 2 \mathrm{H}, \mathrm{ArH}), 7.36 \sim 7.49(\mathrm{~m}, 6 \mathrm{H}, \mathrm{ArH})$, 8.84 (br, 2H, 2NH), 11.32 (br, 1H, NH); ${ }^{13} \mathrm{C}$ HMR $(75$ MHz, DMSO- $\left.d_{6}\right) \delta: 26.7,26.7,44.9,44.9,93.8,114.8$, $115.1,120.5$ (d, $J=7.5 \mathrm{~Hz}), 127.5,129.3,133.3,136.3$, 142.4, 157.3 (d, $J=237.0 \mathrm{~Hz}), 167.4,168.9,186.5$; IR (KBr) $v$ : 3426, 3307, 3040, 2947, 1785, 1627, 1603, 1570, $1507,1485,1439,1346,1284,1207,1159,1090,1034$, $878,834,791,722 \mathrm{~cm}^{-1}$; HRMS (TOF $\mathrm{ES}^{+}$) calcd for $\mathrm{C}_{20} \mathrm{H}_{19} \mathrm{ClFN}_{3} \mathrm{O}_{2}[\mathrm{M}+\mathrm{H}]^{+}$388.1223, found 388.1224.

3-(4-氯苯基)-2-(1,3-安定-2-亚基)-3-氧代- $N$-[4-(三 氟甲基)苯基]丙酰胺 $(\mathbf{6 g})$ : 得 $407 \mathrm{mg}$, 白色固体, 产率 93\%. m.p. 236.2 238.2 ${ }^{\circ} \mathrm{C} ;{ }^{1} \mathrm{H}$ NMR $(300 \mathrm{MHz}$, DMSO- $\left.d_{6}\right) \delta: 1.51 \sim 1.55\left(\mathrm{~m}, 4 \mathrm{H}, 2 \mathrm{CH}_{2}\right), 2.93 \sim 2.97(\mathrm{~m}$, $\left.4 \mathrm{H}, 2 \mathrm{CH}_{2}\right), 7.38 \sim 7.42(\mathrm{~m}, 4 \mathrm{H}, \mathrm{ArH}), 7.57(\mathrm{~d}, J=8.7 \mathrm{~Hz}$, $2 \mathrm{H}, \mathrm{ArH}), 7.72(\mathrm{~d}, J=8.4 \mathrm{~Hz}, 2 \mathrm{H}, \mathrm{ArH}), 8.75(\mathrm{br}, 2 \mathrm{H}$, 2NH), 12.08 (br, $1 \mathrm{H}, \mathrm{NH}) ;{ }^{13} \mathrm{C}$ HMR (75 MHz, DMSO- $d_{6}$ ) $\delta: 26.5,26.5,45.0,45.0,94.3,118.5,121.4 \sim 122.8(\mathrm{~m})$, $125.8,127.6,129.1,133.3,142.4,143.8,167.3,169.0$, 186.2; IR (KBr) v: 3425, 3315, 3048, 2950, 1781, 1633, 1593, 1575, 1489, 1410, 1362, 1281, 1159, 1108, 1067, 1013, 841, 786, $720 \mathrm{~cm}^{-1}$; HRMS (TOF $\mathrm{ES}^{+}$) calcd for $\mathrm{C}_{21} \mathrm{H}_{19} \mathrm{ClF}_{3} \mathrm{~N}_{3} \mathrm{O}_{2}[\mathrm{M}+\mathrm{H}]^{+}, 438.1191$, found 438.1192.

\section{3 化合物 7 的合成方法}

称取 $1 \mathrm{mmol}$ 杂环烯酮缩胺 3 加入到 $25 \mathrm{~mL}$ 圆底烧 瓶中, 加入 $15 \mathrm{~mL}$ 溶剂搅拌溶解, 并在摚拌下缓慢加入 $1.1 \mathrm{mmol}$ 苯基异氭酸 4. 充分搅拌后加入 $0.5 \mathrm{mmol}$ $\mathrm{Cs}_{2} \mathrm{CO}_{3}$, 室温下搅拌 $2.5 \mathrm{~h}, \mathrm{TLC}$ 监测直至杂环烯酮缩胺 反应完全后停止反应. 加水淬灭反应时析出固体，搅拌 $10 \mathrm{~min}$ 左右后静置 $0.5 \mathrm{~h}$ 后抽滤. 抽滤所得固体用适量 丙酮重结晶得目标产物 7.

(E)- $N$-(4-氯苯基)-2-(2-氧代-2-苯基亚乙基)咪唑烷1-甲酰胺 (7a): 得 $311 \mathrm{mg}$, 白色固体, 产率 91\%. m.p. $142.3 \sim 144.3{ }^{\circ} \mathrm{C}$; ${ }^{1} \mathrm{H}$ NMR (500 MHz, DMSO- $d_{6}$ ) $\delta: 3.74$ (t, $\left.J=8.4 \mathrm{~Hz}, 2 \mathrm{H}, \mathrm{CH}_{2}\right), 4.10\left(\mathrm{t}, J=8.4 \mathrm{~Hz}, 2 \mathrm{H}, \mathrm{CH}_{2}\right), 6.76$ (s, $1 \mathrm{H}, \mathrm{CH}), 7.39 \sim 7.44(\mathrm{~m}, 5 \mathrm{H}, \mathrm{ArH}), 7.60(\mathrm{~d}, J=8.7 \mathrm{~Hz}$, 2H, ArH), 7.78 (t, $J=3.7 \mathrm{~Hz}, 2 \mathrm{H}, \mathrm{ArH}), 9.09$ (br, 1H, NH), 
10.33 (br, $1 \mathrm{H}, \mathrm{NH}) ;{ }^{13} \mathrm{C}$ HMR (125 MHz, DMSO- $\left.d_{6}\right) \delta$ : 42.1, 45.4, 78.5, 122.9, 126.8, 127.6, 128.6, 128.8, 130.6, 138.0, 141.1, 151.5, 160.0, 185.8; IR (KBr) v: 3434, 2925, 2855, 16998, 1599, 1577, 1494, 1439, 1397, 1317, 1239, 1176, 1094, 1059, 907, 826, 726, 696, 648, $507 \mathrm{~cm}^{-1}$; HRMS (TOF ES ${ }^{+}$) calcd for $\mathrm{C}_{18} \mathrm{H}_{15} \mathrm{ClN}_{3} \mathrm{O}_{2}[\mathrm{M}-\mathrm{H}]^{-}$ 340.0858 , found 340.0859 .

(E)- $N$-(4-氯苯基)-2-[2-(4-甲氧基苯基)-2-氧代亚乙 基]咪唑烷-1-甲酰胺(7b): 得 $334 \mathrm{mg}$, 白色固体, 产率 90\%. m.p. 158.6 160.0 ${ }^{\circ} \mathrm{C} ;{ }^{1} \mathrm{H}$ NMR (300 MHz, DMSO$\left.d_{6}\right) \delta: 3.71\left(\mathrm{t}, J=8.1 \mathrm{~Hz}, 2 \mathrm{H}, \mathrm{CH}_{2}\right), 3.79\left(\mathrm{~s}, 3 \mathrm{H}, \mathrm{OCH}_{3}\right)$, $4.08\left(\mathrm{t}, J=8.3 \mathrm{~Hz}, 2 \mathrm{H}, \mathrm{CH}_{2}\right), 6.72(\mathrm{~s}, 1 \mathrm{H}, \mathrm{CH}), 6.97$ (d, $J=8.7 \mathrm{~Hz}, 2 \mathrm{H}, \mathrm{ArH}), 7.39$ (d, $J=8.7 \mathrm{~Hz}, 2 \mathrm{H}, \mathrm{ArH}), 7.60$ (d, $J=8.7 \mathrm{~Hz}, 2 \mathrm{H}, \mathrm{ArH}), 7.75$ (d, $J=8.4 \mathrm{~Hz}, 2 \mathrm{H}, \mathrm{ArH})$, 9.06 (br, 1H, NH), 10.26 (br, 1H, ArNH); ${ }^{13} \mathrm{C}$ HMR (75 $\left.\mathrm{MHz}, \mathrm{DMSO}-d_{6}\right) \delta: 41.7,45.0,55.2,77.5,113.4,122.5$, $127.1,128.2$, 128.4, 133.2, 137.6, 151.1, 159.2, 161.0, 184.8; IR (KBr) v: 3429, 2895, 2838, 1702, 1598, 1575, 1489, 1392, 1319, 1243, 1169, 1094, 1060, 1030, 966, 906, 827, 776, 708, 604, $508 \mathrm{~cm}^{-1}$; HRMS (TOF ES ${ }^{+}$) calcd for $\mathrm{C}_{19} \mathrm{H}_{17} \mathrm{ClN}_{3} \mathrm{O}_{3}[\mathrm{M}-\mathrm{H}]^{-}, 370.0963$, found 370.0964 .

(E)- $N$-(4-氯苯基)-2-[2-氧代-2-(对甲苯基)亚乙基]咪 唑烷-1-甲酰胺(7c): 得 $316 \mathrm{mg}$, 白色固体, 产率 $89 \%$. m.p. $194.0 \sim 196.0{ }^{\circ} \mathrm{C} ;{ }^{1} \mathrm{H}$ NMR (300 MHz, DMSO- $\left.d_{6}\right) \delta$ : $2.33\left(\mathrm{~s}, 3 \mathrm{H}, \mathrm{CH}_{3}\right), 3.69 \sim 3.75\left(\mathrm{~m}, 2 \mathrm{H}, \mathrm{CH}_{2}\right), 4.05 \sim 4.09$ $\left(\mathrm{m}, 2 \mathrm{H}, \mathrm{CH}_{2}\right), 6.74$ (s, 1H, CH), 7.23 (d, J=7.2 Hz, 2H, ArH), 7.39 (d, $J=8.1 \mathrm{~Hz}, 2 \mathrm{H}, \operatorname{ArH}), 7.60$ (d, $J=7.8 \mathrm{~Hz}$, 2H, ArH), 7.68 (d, J=7.2 Hz, 2H, ArH), 9.07 (br, 1H, $\mathrm{NH}), 10.30$ (br, $1 \mathrm{H}, \mathrm{NH}) ;{ }^{13} \mathrm{C}$ HMR (75 MHz, DMSO- $d_{6}$ ) $\delta: 20.9,41.7,45.0,77.8,122.5,126.4,127.1,128.4,128.7$, 137.8 (d, $J=22.5 \mathrm{~Hz}) 140.0,151.1,159.4,185.2$; IR (KBr) $v: 3303,3138,3106,2975,2942,2898,1690,1599,1571$, $1491,1476,1392,1353,1313,1283,1244,1091,1057$, 1017, 903, 854, 826, 771, 752, 550, $503 \mathrm{~cm}^{-1}$; HRMS $\left(\mathrm{TOF} \mathrm{ES}^{+}\right.$) calcd for $\mathrm{C}_{19} \mathrm{H}_{17} \mathrm{ClN}_{3} \mathrm{O}_{2}[\mathrm{M}-\mathrm{H}]^{-} 354.1014$, found 354.1011 .

(E)- $N$-(4-氯苯基)-2-[2-(4-氟苯基)-2-氧代亚乙基]咪 唑烷-1-甲酰胺(7d): 得 $305 \mathrm{mg}$, 白色固体, 产率 $85 \%$. m.p. $157.4 \sim 159.2{ }^{\circ} \mathrm{C} ;{ }^{1} \mathrm{H}$ NMR $\left(300 \mathrm{MHz}, \mathrm{DMSO}-d_{6}\right) \delta$ : $3.73\left(\mathrm{t}, J=8.3 \mathrm{~Hz}, 2 \mathrm{H}, \mathrm{CH}_{2}\right), 4.10$ (t, $J=8.4 \mathrm{~Hz}, 2 \mathrm{H}, \mathrm{CH}_{2}$ ), $6.73(\mathrm{~s}, 1 \mathrm{H}, \mathrm{CH}), 7.21 \sim 7.27(\mathrm{~m}, 2 \mathrm{H}, \mathrm{ArH}), 7.39(\mathrm{~d}, J=$ $8.7 \mathrm{~Hz}, 2 \mathrm{H}, \mathrm{ArH}), 7.60$ (d, $J=8.7 \mathrm{~Hz}, 2 \mathrm{H}, \mathrm{ArH}), 7.81 \sim$ 7.86 (m, 2H, ArH), 9.09 (br, 1H, NH), 10.28 (br, 1H, NH); ${ }^{13} \mathrm{C}$ HMR (75 MHz, DMSO-d $\left.d_{6}\right) \delta: 41.7,45.0,77.7,115.0$ (d, $J=21.0 \mathrm{~Hz}), 122.5,127.2,128.4,128.8$ (d, $J=9.0$
$\mathrm{Hz}), 137.1,137.6,151.1,159.6,183.9$; IR (KBr) v: 3425, 2975, 2901, 1691, 1598, 1510, 1491, 1401, 1317, 1285, 1244, 1155, 1093, 1058, 1013, 906, 828, 775, 597, 505 $\mathrm{cm}^{-1}$; HRMS (TOF ES ${ }^{+}$) calcd for $\mathrm{C}_{18} \mathrm{H}_{14} \mathrm{ClFN}_{3} \mathrm{O}_{2}[\mathrm{M}-$ $\mathrm{H}]^{-}$358.0764, found 358.0761 .

(E)- $N$-(4-氯苯基)-2-[2-(4-氯苯基)-2-氧代亚乙基]咪 唑烷-1-甲酰胺(7e): 得 $327 \mathrm{mg}$, 白色固体, 产率 $87 \%$. m.p. 192.7 $194.7{ }^{\circ} \mathrm{C} ;{ }^{1} \mathrm{H}$ NMR (300 MHz, DMSO-d 6 ) $\delta$ : $3.70 \sim 3.76\left(\mathrm{~m}, 2 \mathrm{H}, \mathrm{CH}_{2}\right), 4.06 \sim 4.12\left(\mathrm{~m}, 2 \mathrm{H}, \mathrm{CH}_{2}\right), 6.74$ (s, 1H, CH), 7.39 (d, $J=8.1 \mathrm{~Hz}, 2 \mathrm{H}, \mathrm{ArH}), 7.48$ (d, $J=7.5$ $\mathrm{Hz}, 2 \mathrm{H}, \mathrm{ArH}), 7.60$ (d, $J=7.8 \mathrm{~Hz}, 2 \mathrm{H}, \mathrm{ArH}), 7.79$ (d, $J=$ $7.5 \mathrm{~Hz}, 2 \mathrm{H}, \mathrm{ArH}$ ), 9.09 (br, 1H, NH), 10.32 (br, 1H, ArNH); ${ }^{13} \mathrm{C}$ HMR (75 MHz, DMSO- $d_{6}$ ) $\delta: 41.8,45.0,77.9$, $122.5,127.2,128.2,128.2,128.4,134.9,137.5,139.3$, 151.0, 159.8, 183.7; IR (KBr) v: 3310, 3140, 2974, 2897, $1689,1601,1570,1494,1474,1399,1313,1281,1245$, 1176, 1088, 1057, 1029, 1014, 964, 903, 879, 854, 825, 772, 754, 683, 634, 549, $502 \mathrm{~cm}^{-1}$; HRMS (TOF ES ${ }^{+}$) calcd for $\mathrm{C}_{18} \mathrm{H}_{14} \mathrm{Cl}_{2} \mathrm{~N}_{3} \mathrm{O}_{2}[\mathrm{M}-\mathrm{H}]^{-}$374.0469, found 374.0469 .

(E)-2-[2-(4-溴苯基)-2-氧代亚乙基]- $N$-(4-氯苯基)咪 唑烷-1-甲酰胺(7f): 得 $328 \mathrm{mg}$, 白色固体, 产率 78\%. m.p. $251.3 \sim 253.3{ }^{\circ} \mathrm{C} ;{ }^{1} \mathrm{H}$ NMR (300 MHz, DMSO- $\left.d_{6}\right) \delta$ : $3.70 \sim 3.76\left(\mathrm{~m}, 2 \mathrm{H}, \mathrm{CH}_{2}\right), 4.06 \sim 4.12\left(\mathrm{~m}, 2 \mathrm{H}, \mathrm{CH}_{2}\right), 6.74$ (s, 1H, CH), $7.37 \sim 7.43(\mathrm{~m}, 2 \mathrm{H}, \mathrm{ArH}), 7.57 \sim 7.63(\mathrm{~m}, 4 \mathrm{H}$, ArH), $7.67 \sim 7.73$ (m, 2H, ArH), 9.09 (br, 1H, NH), 10.32 (br, $1 \mathrm{H}, \mathrm{NH}) ;{ }^{13} \mathrm{C}$ HMR (75 MHz, DMSO- $\left.d_{6}\right) \delta: 41.8,45.0$, 77.8, 122.5, 123.8, 127.2, 128.4, 128.5, 131.2, 137.5, 139.7, 151.0, 159.8, 183.8; IR (KBr) v: 3435, 3308, 3140, 2974, 2896, 1689, 1630, 1600, 1567, 1493, 1473, 1399, 1312, 1244, 1176, 1091, 1069, 1054, 1010, 903, 878, 851, 824, 771, 752, 634, 542, $502 \mathrm{~cm}^{-1}$; HRMS (TOF ES ${ }^{+}$) calcd for $\mathrm{C}_{18} \mathrm{H}_{14} \mathrm{BrClN}_{3} \mathrm{O}_{2}[\mathrm{M}-\mathrm{H}]^{-}$417.9963, found 417.9965.

(E)- $N$-(4-氯苯基)-2-[2-(2-氯苯基)-2-氧代亚乙基]咪 唑烷-1-甲酰胺 $(7 \mathbf{g})$ : 得 $297 \mathrm{mg}$, 白色固体, 产率 79\%. m.p. $170.1 \sim 172.1{ }^{\circ} \mathrm{C} ;{ }^{1} \mathrm{H}$ NMR (300 MHz, DMSO- $\left.d_{6}\right) \delta$ : $3.74\left(\mathrm{t}, J=8.4 \mathrm{~Hz}, 2 \mathrm{H}, \mathrm{CH}_{2}\right), 4.09\left(\mathrm{t}, J=8.6 \mathrm{~Hz}, 2 \mathrm{H}, \mathrm{CH}_{2}\right)$, $6.21(\mathrm{~s}, 1 \mathrm{H}, \mathrm{CH}), 7.33 \sim 7.44(\mathrm{~m}, 6 \mathrm{H}, \mathrm{ArH}), 7.54(\mathrm{~d}, J=$ $9.0 \mathrm{~Hz}, 2 \mathrm{H}, \mathrm{ArH}), 9.08$ (br, 1H, NH), 10.09 (br, 1H, NH); ${ }^{13} \mathrm{C}$ HMR (75 MHz, DMSO- $\left.d_{6}\right) \delta: 41.9,44.9,82.0,122.4$, $126.9,127.2$, 128.4, 128.6, 129.4, 129.7, 129.8, 137.5, $142.4,150.9,159.0,186.6$; IR (KBr) v: 3259, 3187, 3118, 3063, 2977, 2897, 1706, 1601, 1564, 1543, 1490, 1396, $1317,1239,1177,1094,1074,1045,1013,968,907,828$, 
750, 688, 637, $509 \mathrm{~cm}^{-1}$; HRMS (TOF ES ${ }^{+}$) calcd for $\mathrm{C}_{18} \mathrm{H}_{14} \mathrm{Cl}_{2} \mathrm{~N}_{3} \mathrm{O}_{2}[\mathrm{M}-\mathrm{H}]^{-}$374.0469, found 374.0468.

(E)- $N$-(4-氯苯基)-2-[2-氧代-2-(噻吩-2-基)亚乙基]咪 唑烷-1-甲酰胺(7h): 得 $316 \mathrm{mg}$, 黄色固体, 产率 91\%. m.p. 180.4 182.4 ${ }^{\circ} \mathrm{C} ;{ }^{1} \mathrm{H}$ NMR (300 MHz, DMSO- $d_{6}$ ) $\delta$ : $3.68 \sim 3.74\left(\mathrm{~m}, 2 \mathrm{H}, \mathrm{CH}_{2}\right), 4.05 \sim 4.09\left(\mathrm{~m}, 2 \mathrm{H}, \mathrm{CH}_{2}\right), 6.63$ (s, $1 \mathrm{H}, \mathrm{CH}), 7.07 \sim 7.11(\mathrm{~m}, 1 \mathrm{H}, \mathrm{ArH}), 7.40 \sim 7.46(\mathrm{~m}, 3 \mathrm{H}$, ArH), $7.57 \sim 7.65$ (m, 3H, ArH), 9.09 (br, 1H, NH), 10.00 (br, $1 \mathrm{H}, \mathrm{NH}) ;{ }^{13} \mathrm{C}$ HMR (75 MHz, DMSO- $\left.d_{6}\right) \delta: 41.8,45.0$, $77.8,122.5,126.6,127.2,127.9,128.4,129.7,137.5$, 147.8, 151.0, 159.1, 178.8; IR (KBr) v: 3378, 3152, 3101, 3061, 2977, 2890, 1697, 1594, 1491, 1421, 1399, 1318, 1279, 1250, 1231, 1090, 1075, 1058, 1035, 1010, 967, 871, 853, 829, 772, 714, 613, $509 \mathrm{~cm}^{-1}$; HRMS (TOF ES ${ }^{+}$) calcd for $\mathrm{C}_{16} \mathrm{H}_{13} \mathrm{ClN}_{3} \mathrm{O}_{2} \mathrm{~S}[\mathrm{M}-\mathrm{H}]^{-}$346.0422, found 346.0422 .

(E)-N-(4-氯苯基)-2-(硝基亚甲基)咪唑烷-1-甲酰胺 (7i): 得 $254 \mathrm{mg}$, 黄色固体, 产率 90\%. m.p. 170.2 $172.2{ }^{\circ} \mathrm{C} ;{ }^{1} \mathrm{H}$ NMR (300 MHz, DMSO- $\left.d_{6}\right) \delta: 3.76(\mathrm{t}, J=$ $\left.8.4 \mathrm{~Hz}, 2 \mathrm{H}, \mathrm{CH}_{2}\right), 4.15$ (t, $\left.J=8.6 \mathrm{~Hz}, 2 \mathrm{H}, \mathrm{CH}_{2}\right), 7.39$ (d, $J=8.4 \mathrm{~Hz}, 2 \mathrm{H}, \mathrm{ArH}), 7.54$ (d, $J=8.4 \mathrm{~Hz}, 2 \mathrm{H}, \mathrm{ArH}), 7.66$ (s, 1H, CH), 9.17 (br, 1H, NH), $9.70(\mathrm{br}, 1 \mathrm{H}, \mathrm{NH}) ;{ }^{13} \mathrm{C}$ HMR (75 MHz, DMSO- $d_{6}$ ) $\delta$ : 42.5, 45.7, 100.8, 122.8, 127.6, 128.4, 137.1, 150.5, 155.4; IR (KBr) v: 3300, 3179, 3108, 3054, 2981, 2906, 1707, 1597, 1538, 1488, 1461, 1420, 1382, 1321, 1240, 1215, 1095, 1075, 1013, 995, 946, 833, 807, 790, 766, 743, 594, $506 \mathrm{~cm}^{-1}$; HRMS (TOF $\mathrm{ES}^{+}$) calcd for $\mathrm{C}_{11} \mathrm{H}_{10} \mathrm{ClN}_{4} \mathrm{O}_{3}[\mathrm{M}-\mathrm{H}]^{-}$281.0446, found 281.0445 .

(E)-2-(2-氧代-2-苯基亚乙基)- $N$-(对甲苯基)味唑烷1-甲酰胺 $(7 \mathbf{j})$ : 得 $273 \mathrm{mg}$, 白色固体, 产率 $85 \%$. m.p. $175.1 \sim 177.1{ }^{\circ} \mathrm{C} ;{ }^{1} \mathrm{H}$ NMR (300 MHz, DMSO- $d_{6}$ ) $\delta: 2.27$ (s, 3H, $\mathrm{CH}_{3}$ ), 3.72 (t, $\left.J=8.4 \mathrm{~Hz}, 2 \mathrm{H}, \mathrm{CH}_{2}\right), 4.08$ (t, $J=8.5$ $\left.\mathrm{Hz}, 2 \mathrm{H}, \mathrm{CH}_{2}\right), 6.75(\mathrm{~s}, 1 \mathrm{H}, \mathrm{CH}), 7.14(\mathrm{~d}, J=8.1 \mathrm{~Hz}, 2 \mathrm{H}$, $\mathrm{ArH}), 7.41 \sim 7.44$ (m, 5H, ArH), $7.76 \sim 7.80(\mathrm{~m}, 2 \mathrm{H}, \mathrm{ArH})$, $8.91(\mathrm{br}, 1 \mathrm{H}, \mathrm{NH}), 10.34$ (br, $1 \mathrm{H}, \mathrm{NH}) ;{ }^{13} \mathrm{C}$ HMR $(75 \mathrm{MHz}$, DMSO- $\left.d_{6}\right) \delta: 20.4,41.7,45.0,77.9,121.2,126.4,128.2$, $128.9,130.2,132.5,135.9,140.7,151.2,159.8,185.2$; IR (KBr) $v$ : 3422, 3328, 3085, 3042, 2976, 2900, 1782, 1687, 1599, 1576, 1496, 1439, 1396, 1318, 1243, 1105, 1023, 814, 743, $727 \mathrm{~cm}^{-1}$; HRMS (TOF $\mathrm{ES}^{+}$) calcd for $\mathrm{C}_{19} \mathrm{H}_{19} \mathrm{~N}_{3} \mathrm{O}_{2}[\mathrm{M}+\mathrm{H}]^{+}$322.1550, found 322.1555 .

( $E$ )- $N$-(4-氟苯基)-2-(2-氧代-2-苯基亚乙基)咪唑烷1-甲酰胺 (7k): 得 $296 \mathrm{mg}$, 白色固体, 产率 $91 \%$. m.p. $165.8 \sim 167.8{ }^{\circ} \mathrm{C} ;{ }^{1} \mathrm{H}$ NMR (300 MHz, DMSO- $d_{6}$ ) $\delta: 3.73$ (t, $J=8.4 \mathrm{~Hz}, 2 \mathrm{H}, \mathrm{CH}_{2}$ ), 4.09 (t, $J=8.4 \mathrm{~Hz}, 2 \mathrm{H}, \mathrm{CH}_{2}$ ), 6.77 (s, $1 \mathrm{H}, \mathrm{CH}), 7.18(\mathrm{~d}, J=8.9 \mathrm{~Hz}, 2 \mathrm{H}, \mathrm{ArH}), 7.42 \sim 7.44(\mathrm{~m}$, $3 \mathrm{H}, \mathrm{ArH}), 7.54 \sim 7.58(\mathrm{~m}, 2 \mathrm{H}, \mathrm{ArH}), 7.77 \sim 7.80(\mathrm{~m}, 2 \mathrm{H}$, ArH), 9.02 (br, 1H, NH), 10.34 (br, $1 \mathrm{H}, \mathrm{NH}$ ); ${ }^{13} \mathrm{C} \mathrm{HMR}$ (75 MHz, DMSO- $d_{6}$ ) $\delta: 41.7,45.0,78.0,114.9,115.2$, $123.2(\mathrm{~d}, J=7.5 \mathrm{~Hz}), 126.4,127.9,128.2,130.2,134.8$, 140.7, 151.3, 158.4 (d, $J=238.5 \mathrm{~Hz}), 159.7,185.4$; IR (KBr) $v$ : 3421, 3295, 3076, 2976, 2900, 1690, 1599, 1577, $1500,1480,1405,1316,1253,1177,1058,831,802,780$, $747,728 \mathrm{~cm}^{-1}$; HRMS (TOF ES ${ }^{+}$) calcd for $\mathrm{C}_{18} \mathrm{H}_{16} \mathrm{FN}_{3} \mathrm{O}_{2}$ $[\mathrm{M}+\mathrm{H}]^{+}$326.1299, found 326.1302.

(E)-2-(2-氧代-2-苯基亚乙基)- $N$-[4-(三氟甲基)苯基] 咪唑烷-1-甲酰胺(7l): 得 $349 \mathrm{mg}$, 白色固体, 产率 93\%. m.p. $154.2 \sim 156.2{ }^{\circ} \mathrm{C} ;{ }^{1} \mathrm{H}$ NMR (300 MHz, DMSO- $\left.d_{6}\right) \delta$ : 3.75 (t, $\left.J=8.3 \mathrm{~Hz}, 2 \mathrm{H}, \mathrm{CH}_{2}\right), 4.14\left(\mathrm{t}, J=8.4 \mathrm{~Hz}, 2 \mathrm{H}, \mathrm{CH}_{2}\right)$, $6.78(\mathrm{~s}, 1 \mathrm{H}, \mathrm{CH}), 7.42 \sim 7.45(\mathrm{~m}, 3 \mathrm{H}, \mathrm{ArH}), 7.69 \sim 7.84$ (m, 6H, ArH), 9.31 (br, 1H, NH), $10.36(\mathrm{br}, 1 \mathrm{H}, \mathrm{NH}) ;{ }^{13} \mathrm{C}$ HMR $\left(75 \mathrm{MHz}\right.$, DMSO- $\left.d_{6}\right) \delta: 41.8,45.0,78.1,120.4$, $122.6 \sim 123.5(\mathrm{~m}), 125.7,126.4,128.2,130.3,140.6$, 142.5, 151.0, 159.5, 185.5; IR (KBr) v: 3423, 3282, 3061, 2951, 2898, 1606, 1578, 1500,1485, 1440, 1322, 1244, 1115, 1067, 1031, 1019, 840, 801, 746, 724, $\mathrm{cm}^{-1}$; HRMS $\left(\right.$ TOF $\mathrm{ES}^{+}$) calcd for $\mathrm{C}_{19} \mathrm{H}_{16} \mathrm{~F}_{3} \mathrm{~N}_{3} \mathrm{O}_{2}[\mathrm{M}+\mathrm{H}]^{+}$376.1267, found 376.1270 .

辅助材料(Supporting Information) 化合物的 ${ }^{1} \mathrm{H}$ NMR 和 ${ }^{13} \mathrm{C}$ NMR 谱图. 这些材料可以免费从本刊网站 (http://sioc-journal.cn/)上下载.

\section{References}

[1] (a) Batra, S.; Tusi, Z.; Madappaa, S. Curr. Med. Chem.: Anti-Infect. Agents 2006, 5, 135.

(b) He, X.; Zhong, M.; Yang, J.; Wu, Z.; Xiao, Y.; Guo, H.; Hu, X. Chem. Biol. Drug Des. 2012, 79, 771.

(c) Li, H.-Q.; Zhu, T.-T.; Yan, T.; Luo, Y.; Zhu, H.-L. Eur. J. Med. Chem. 2009, 44, 453.

[2] (a) Nourry, A.; Zambon, A.; Davies, L.; Niculescu-Duvaz, I.; Dijkstra, H. P.; Ménard, D.; Gaulon, C.; Niculescu-Duvaz, D.; Suijkerbuijk, B. M.; Friedlos, F.; Manne, H. A.; Kirk, R.; Whittaker, S.; Marais, R.; Springer, C. J. J. Med. Chem. 2010, 53, 1964.

(b) Ménard, D.; Niculescu-Duvaz, I.; Dijkstra, H. P.; NiculescuDuvaz, D.; Suijkerbuijk, B. M.; Zambon, A.; Nourry, A.; Roman, E.; Davies, L.; Manne, H. A.; Friedlos, F.; Kirk, R.; Whittaker, S.; Gill, A.; Taylor, R. D.; Marais, R.; Springer, C. J. J. Med. Chem. 2009, 52, 3881.

(c) Reddy, N. V.; Kumar, P. S.; Reddy, P. S.; Kantam, M. L.; Reddy, K. R. New J. Chem. 2015, 39, 805.

(d) Wang, W.-L.; Luo, H.; Gao, Y.; Gao, L.-X.; Cheng, L.; Zhou, Y.-B.; Li, J.; Li, J.-Y.; Feng, B.-N. 2016, 36, 2142 (in Chinese). (王文龙, 骆欢, 高雅, 高立信, 盛丽, 周宇波, 李佳, 李静雅, 冯柏年, 有机化学, 2016, 36, 2142.) 
[3] (a) Yu, S.; Haight, A.; Kotecki, B.; Wang, L.; Lukin, K.; Hill, D. H. J. Org. Chem. 2009, 74, 9539.

(b) Clayden, J.; Hennecke, U. Org. Lett. 2008, 10, 3567.

(c) Clayden, J.; Dufour, J.; Grainger, D. M.; Helliwell, M. J. Am. Chem. Soc. 2007, 129, 7488 .

(d) Gao, J.; Li, H.; Zhang, Y.; Zhang, Y. Green Chem. 2007, 9, 572.

(e) Sheng, G..; Zhang, W.; Chin. J. Org. Chem. 2013, 33, 2271 (in Chinese).

(盛国柱, 张炜, 有机化学, 2013, 33, 2271.)

(f) Yang, R.; Zhao, Y.; Jiang, M.; Yan, S.; Lin, J. Chin. J. Org. Chem. 2016, 36, 2941 (in Chinese).

(杨瑞霞, 赵宇澄, 蒋美妤, 严胜骄, 林军, 有机化学, 2016, 36, 2941.)

[4] (a) Gallou, I. Org. Prep. Proced. Int. 2007, 4, 355.

(b) Bigi, F.;Maggi, R.; Sartori, G. Green Chem. 2000, 2, 140.

(c) Zhang, Z.; Schreiner, P. R. Chem. Soc. Rev. 2009, 38, 1187.

(d) Wang, H.; Zhao, W. Chin. J. Org. Chem. 2013, 33, 1822 (in Chinese).

(王宏社, 赵卫星, 有机化学, 2013, 33, 1822.)

[5] (a) Fabio, R. D.; Griffante, C.; Alvaro, G.; Pentassuglia, G.; Pizzi, D. A.; Donati, D.; Rossi, T.; Guercio, G.; Mattioli, M.; Cimarosti, Z.; Marchioro, C.; Provera, S.; Zonzini, L.; Montanari, D.; Melotto, S.; Gerrard, P. A.; Trist, D. G.; Ratti, E.; Corsi, M. J. Med. Chem. 2009, 52, 3238 .

(b) Cao, P.; Huang, X.-F.; Ding, H.; Ge, H.-M.; Li, H.-Q.; Ruan, B.-F.; Zhu, H.-L. Chem. Biodiversity 2007, 4, 881.

(c) Debnath, A. K. J. Med. Chem.1999, 42, 249.

(d) Zhang, X.; Rodrigues, J.; Evans, L.; Hinkle, B.; Ballantyne, L.; Pena, M. J. Org. Chem. 1997, 62, 6420.

[6] Loto, R. T.; Loto, C. A.; Popoola, A. P. I. J. Mater. Environ. Sci. 2012, 3, 885.

[7] Bucha, H. C.; Todd, C. W. Science 1951, 114, 493.

[8] Bigi, F.; Maggi, R.; Sartori, G.; Zambonin, E. Chem. Commun. 1998, 4, 513.

[9] (a) Li, M.; Shao, P.; Wang, S.-W.; Kong, W.; Wen, L.-R. J. Org. Chem. 2012, 77, 8956.

(b) Yu, F.-C.; Huang, R.; Ni, X.-C.; Fan, J.; Yan, S.-J.; Lin, J. Green Chem. 2013, 15, 453.

(c) Yaqub, M.; Arif, N.; Perveen, R.; Batool1, J.; Riaz, M. T.; Yaseen, M. Asian J. Org. Chem. 2015, 27, 1013.

(d) Zhou, B.; Liu, Z.-C.; Qu, W.-W.; Yang, R.; Lin, X.-R.; Yan, S.-J.; Lin, J. Green Chem. 2014, 16, 4359.

(e) Xu, W.-Y.; Jia, Y.-M.; Yang, J.-K.; Huang, Z.-T.; Yu, C.-Y. Sylett 2010, 1682.

(f) Kong, L.; Yang, R.; Du, X.; Yan, S.; Lin, J. Chin. J. Org. Chem. 2016, 36, 1994 (in Chinese).

(孔令斌, 杨瑞霞, 杜璇璇, 严胜骄, 林军, 有机化学, 2016, 36, 1994.)

[10] (a) Wang, K.-M.; Yan, S.-J.; Lin, J. Eur. J. Chem. 2014, 1129.

(b) Zheng, C.-C.; Liu, F.-J.; Ping, D.-W.; Hu, L.-M.; Cai, Y.-L.; Zhong, R. G. J. Org. Chem. 2009, 74, 6386.

(c) Chen, X.-B.; Liu, Z.-C.; Yang, L.-F.; Yan, S.-J.; Lin, J. ACS Sustainable Chem. Eng. 2014, 2, 1155.

(d) Chen, X.-B.; Liu, Z.-C.; Lin, X.-R.; Huang, R.; Yan, S.-J.; Lin, J. ACS Sustainable Chem. Eng. 2014, 2, 2391.

(e) Peng, M.; Yang, R.; Liu, X.; Yan, S.; Lin, J. Chin. J. Org. Chem. 2015, 35, 1754 (in Chinese).
(彭美阳, 杨瑞霞, 刘昔敏, 严胜骄, 林军, 有机化学, 2015, 35, 1754.)

[11] (a) Yu, F.-C.; Yan, S.-J.; Hu, L.; Wang, Y.-C.; Lin, J. Org. Lett. 2011, 13, 4782 .

(b) Yu, F.-C.; Hao, X.-P.; Lin, X.-R.; Yan, S.-J.; Lin, J. Tetrahedron 2015, 71, 4084.

(c) Li, M.; Zhou, Z.-M.; Wen, L.-R.; Qiu, Z.-X. J. Org. Chem. 2011, 76,3054 .

(d) Wen, L.-R.; Li, Z.-R.; Li, M.; Cao, H. Green Chem. 2012, 14, 707.

(e) Wen, L.-R.; Sun, Q.-C.; Zhang, H.-L.; Li, M. Org. Biomol. Chem. 2013, 11, 781 .

[12] (a) Huang, C.; Yan, S.-J.; Zeng, X.-H.; Dai, X.-Y.; Zhang, Y.; Qing, C.; Lin, J. Eur. J. Med. Chem. 2011, 46, 1172.

(b) Yan, S.-J.; Liu, Y.-J.; Chen, Y.-L.; Liu, L.; Lin, J. Bioorg. Med. Chem. Lett. 2010, 20, 5225.

(c) Yan, S.-J.; Huang, C.; Zeng, X.-H.; Huang, R.; Lin, J. Bioorg. Med. Chem. Lett. 2010, 20, 48.

[13] (a) Chen, N.;Meng, X.; Zhu, F.; Cheng, J.; Shao, X.; Li, Z. J. Agric. Food Chem. 2015, 63, 1360.

(b) Shao, X.; Fu, H.; Xu, X.; Xu, X.; Liu, Z.; Li, Z.; Qian, X. J. Agric. Food Chem. 2010, 58, 2696.

[14] Maryanoff, B. E.; Nortey, S. O.; McNally, J. J.; Sanfilippo, P. J.; McComsey, D. F.; Dubinsky, B.; Shank, R. P.; Reitz, A. B. Biomed. Chem. Lett. 1999, 9, 1547.

[15] Abdelhalim, M. M.; El-Saidi, M. M. T.; Rabie, S. T.; Elmegeed, G. A. Steroids 2007, 72, 459.

[16] Wang, M.-X.; Huang, Z.-T. J. Org. Chem. 1995, 60, 2807.

[17] (a) Huang, Z.-T.; Wang, J.-C.; Wang, L.-B. Synth. Commun. 1996, 26, 2285.

(b) Zhao, M.-X.; Wang, M.-X.; Hang, Z.-T. Acta Chim. Sinica 2001, 59, 1763 (in Chinese).

(赵梅欣, 王梅祥, 黄志镗, 化学学报, 2001, 59, 1763.)

(c) Rajappa, S.; Advani, B. G.; Sreenivasan, R. Indian J. Chem. 1977, 15(B), 886.

[18] Yu, C.-Y.; Yan, S.-J.; Zhang, T.; Huang, Z.-T. CN 101041660, 2007 [Chem. Abstr. 2007, 147, 469361].

[19] Liu, B.; Wang, M.-X.; Huang, Z.-T. Synth. Commun. 1999, 29, 4241 .

[20] Baum, K.; Nguyen, N. V.; Gilardi, R.; Flippen-Anderson, J. L.; George, C. J. Org. Chem. 1992, 57, 3026.

[21] Jiang, X.-Y.; Liu, Z.-C.; Fang, L.; Yang, S.-J.; Lin, J. RSC Adv. 2014, 4, 26389.

[22] (a) Wan, J.-P.; Gao, Y. Chem. Rec. 2016, 16, 1164.

(b) Cao, S.; Jing, Y.; Liu, Y.; Wan, J. Chin. J. Org. Chem. 2014, 34, 876 (in Chinese).

(曹硕, 景艳锋, 刘云云, 万结平, 有机化学, 2014, 34, 876.)

[23] Wan, J.-P.; Gan, S.-F.; Sun, G.-L.; Pan, Y.-J. J. Org. Chem. 2009, 74, 2862.

[24] Wan, J.-P.; Lin, Y.; Jing, Y.; Xu, M.; Liu, Y. Tetrahedron 2014, 70, 7874.

[25] Cao, S.; Xin, L.; Liu, Y.; Wan, J.-P.; Wen, C. RSC Adv. 2015, 5, 27372.

[26] (a) Huang, Z.-T.; Wang, M.-X. Synthesis 1992, 1273.

(b) Li, Z.-J.; Charles, D. Synth. Commun. 2001, 31, 527.

(c) Chen, X.-B.; Liu, X.-M.; Huang, R.; Yan, S. J.; Lin, J. Eur. J. Org. Chem. 2013, 4607. 